\title{
Bacterial Swarmers exhibit a Protective Response to Intestinal Stress
}

2 Weijie Chen ${ }^{1,2}$,, Arpan $\mathrm{De}^{1, *}, \mathrm{Hao} \mathrm{Li}^{1, *}$, Justin R. Wright ${ }^{3}$, Regina Lamendella ${ }^{4}$, Dana J. Lukin ${ }^{5}$, 3 Wendy Szymczak ${ }^{6}$, Katherine Sun ${ }^{7}$, Libusha Kelly ${ }^{8}$, Subho Ghosh ${ }^{1}$, Daniel B. Kearns ${ }^{9}$, Zhen He ${ }^{10}$,

$4 \quad \dagger^{\dagger}$, Christian Jobin ${ }^{10}$, Xiaoping Luo ${ }^{1,}$, Arjun Byju ${ }^{1}$, Shirshendu Chatterjee ${ }^{11}$, Beng San Yeoh ${ }^{12,}$, 5 Matam Vijay-Kumar ${ }^{12,}$, Jay X. Tang ${ }^{2}$, Sridhar Mani ${ }^{1}{ }^{1} * *$

$6 \quad{ }^{1}$ Department of Medicine, Genetics and Molecular Pharmacology, Albert Einstein College of 7 Medicine, 1300 Morris Park Avenue, Bronx, NY 10461, USA; ${ }^{2}$ Department of Physics, Brown 8 University, 182 Hope Street, Providence, RI 02912, USA $;{ }^{3}$ Wright Labs, LLC, 419 14th Street, 9 Huntington, PA 16652, USA; ${ }^{4} J u n i a t a$ College, 1700 Moore Street, Huntingdon, PA 16652, 10 USA; ${ }^{5}$ Jill Roberts Center for Inflammatory Bowel Disease, 1283 York Avenue, New York, NY 11 10065, USA; ${ }^{6}$ Clinical Microbiology Laboratory, Montefiore Medical Center, 111 E 210th Street, 12 Bronx, NY 10467, USA; 'Department of Pathology, NYU Langone Health, 560 First Avenue, 13 New York, NY 10016, USA; ${ }^{8}$ Department of Systems \& Computational Biology, and Department 14 of Microbiology \& Immunology, Albert Einstein College of Medicine, 1300 Morris Park Avenue, 15 Bronx, NY 10461, USA; ${ }^{9}$ Department of Biology, Indiana University Bloomington, 107 S. Indiana 16 Avenue, Bloomington, IN 47405, USA; ${ }^{10}$ Department of Medicine, University of Florida, 17 Gainesville, FL 32611, USA; ${ }^{11}$ Department of Mathematics, The City College of New York, 160 18 Convent Avenue, New York, NY 10031, USA; ${ }^{12}$ UT-Microbiome Consortium, Department of 19 Physiology \& Pharmacology, University of Toledo, College of Medicine \& Life Sciences, 3000

20 Transverse Dr, Mail Stop 1008, Toledo, OH 43614, USA

$21 *$ Equal contribution ** Corresponding author

22 E-mail: sridhar.mani@einsteinmed.org

$23 \dagger$ Present address: Department of Colorectal Surgery, The Sixth Affiliated Hospital of Sun Yat-Sen University, 26

24 Yuancun Erheng Road, Tianhe district, Guangzhou, Guangdong, China

$25 \$$ Present address: Institute of Chinese Materia Medica, Shanghai University of Traditional Chinese Medicine, 1200 26 Cailun Road, Shanghai 201203, China

$27 \S$ Present address: Department of Physiology and Pharmacology, University of Toledo, College of Medicine and Life 28 Sciences, Toledo, $\mathrm{OH} 43614$, USA 


\section{Summary}

30 Bacterial swarming, a collective movement on a surface, has rarely been associated with human

31 pathophysiology. Here, we report for the first time that bacterial swarmers are associated with

32 protection against intestinal inflammation. We show that bacterial swarmers are highly predictive

33 of intestinal stress in mice and humans. We isolated a novel Enterobacter swarming strain, SM3,

34 from mouse feces. SM3 and other known commensal swarmers contrast to their respective

35 swarming-deficient, but swimming-competent isogenic strains abrogated intestinal inflammation

36 in mice. Treatment of colitic mice with SM3, but not its mutants, enriched beneficial fecal

37 anaerobes belonging to the family, Bacteroidales S24-7. We observed SM3 swarming associated

38 pathways in the in vivo fecal metatranscriptomes. In vitro growth of S24-7 was enriched in

39 presence of SM3 or its mutants conjecturing that bacterial swarming in vivo might influence SM3's

40 access to S24-7 in the intestines. Overall, our work identifies a new paradigm in which intestinal

41 stress allows for the emergence of swarming bacteria, which can counterintuitively heal intestinal

42 inflammation.

Keywords

47 Bacterial swarmer, intestinal stress, protection, feces, Enterobacter, S24-7. 
Main Text

Introduction

52 Bacterial motility is essential in mucosal colonization and has long been associated with virulence

53 and pathogenesis (Stanton and Savage, 1984; Wiles et al., 2020). Intestinal inflammation, such as

54 inflammatory bowel disease (IBD), is attributed to dysbiosis and the mucosal immune system

55 (Rooks et al., 2014). The disease is characterized by enrichment of flagellated bacteria resident in

56 the microbiome and its encroachment into the inner mucus layer and the intestinal epithelial cells

57 (IEC) (Erben et al., 2014; Okumura et al., 2016; Tran et al., 2019). LY6/PLAUR Domain

58 Containing 8 (LYPD8) (Okada et al., 2020)) is a secreted protein that binds polymerized flagella

59 and prevents bacterial motility on the epithelial surface (Hsu et al., 2017)). Notably, flagellin, ound

60 at high levels during intestinal inflammation (Lodes et al., 2004), is recognized by Toll-like

61 Receptor 5 (TLR5) on IEC. An adaptive immune response is thought to ensue to maintain immune

62 homeostasis (Chassaing et al., 2014a; Chassaing et al., 2014b; Cullender et al., 2013). However,

63 despite these cues, during intestinal health and disease, the functional importance and consequence

64 of bacterial motility in a microbial consortium is unknown.

65 Swimming and swarming are the two primary and common forms of bacterial motility (Kearns,

66 2010). Swarming, driven by flagella, is a fundamental process in certain groups of bacteria

67 characterized by collective and rapid movement across a surface (Be'er and Ariel, 2019; Kearns,

68 2010). This process, in contrast with swimming, offers bacteria a competitive advantage in

69 occupying specific niches (e.g., seeding colonization) (Barak et al., 2009); however, the cost-

70 benefits to bacteria (Butler et al., 2010; Finkelshtein et al., 2015) and consequences to its host or

71 the environment remain primarily unknown (Allison et al., 1994).

72 In the context of intestinal inflammation, we show that bacterial swarmers are a feature of a

73 stressed intestine in mammals. In a mouse model of intestinal stress, bacterial swarmers, when

74 dosed in sufficient abundance, suppressed intestinal inflammation compared to its swarming

75 deficient mutants. We focused on a novel Enterobacter swarming strain SM3, which did not show

76 protection in germ-free mice's acute colitis model. However, we maintained its activity in a TLR5

77 knockout IL-10R antibody-induced mouse model of colitis harboring conventional microflora.

78 SM3 supplementation in mice with acute intestinal inflammation exhibited enrichment of 
79 beneficial anaerobes, specifically the S24-7 group of bacteria, in the feces. SM3 also promoted a

80 bacterial strain belonging to the family S24-7, only when in close interaction, in an in vitro co-

81 culture assay. Swarming deficient strains of SM3 had reduced S24-7 levels in feces. We posit that

82 it is likely the act of swarming in vivo, which facilitates close interaction between SM3 and S24-

83 7, enriches the latter taxa. S24-7 is strongly associated with intestinal healing in mice (Borton et

84 al., 2017; Osaka et al., 2017).

\section{Results}

\section{Presence of Bacterial Swarmers is a feature of a stressed intestine.}

87 To test whether bacterial swarmers are associated with human and rodent gut health, we developed

88 a modified swarming assay using feces based on an established soft-agar plate assay utilized for

89 single species (Morales-Soto et al., 2015). Since prototypical swarming bacteria (e.g., Proteus

90 mirabilis, Pseudomonas aeruginosa) are associated with virulence (Allison et al., 1994; Overhage

91 et al., 2008), we surmised that bacterial swarming might be well represented in colonoscopy

92 samples and feces from humans with bacterial virulence-associated pathologies (e.g., intestinal

93 inflammation)(Yang and Jobin, 2014)). We obtained colonoscopy aspirates from individuals with

94 a progressive illness (inflammatory bowel disease - Crohn's and ulcerative colitis and other

95 common forms of intestinal stress like intestinal polyps (Crespo-Sanjuán et al., 2015; Jass, 2003)

96 as well as age and gender-matched controls (those without a clinically active illness). Within our

97 sampling pool, bacterial collective spreading on soft agar was over-represented in cases with overt

98 or clinically active intestinal stress (Fig. 1a-b). As a preliminary assessment, we judged bacterial

99 swarmers' presence in feces by the bacterial spread with a surfactant layer on soft-agar followed

100 by isolation, identification by MALDI-TOF, and validation of its swarming motility (STAR

101 Method Table 1).

102 In this pilot evaluation, the specificity and positive predictive value of the test for disease as

103 defined was approximately 88 and 89\%, respectively. In comparison, the test's sensitivity and the

104 negative predictive value was only approximately 56 and 52\%, respectively (Fig. 1c). Similarly,

105 feces collected from pigs with active inflammatory bowel disease also showed an increased

106 prevalence of collective spreading and swarming compared to control pigs (Fig. 1d). Despite the

107 caveat that our approach might preclude the selection of swarmers that do not produce surfactant 
108 (Kearns, 2010), these pilot data indicate that collective spreading and swarming is a specific

109 feature, and potentially a biomarker of an intestinal pathology as defined by harboring active

110 intestinal inflammation or polyps.

\section{Novel Enterobacter swarming strains were isolated from mouse feces.}

112 To identify the relevance of swarmers on host health, we focused on isolating endogenous 113 swarming bacteria residing in rodents and humans. An initial approach was to determine if a single 114 dominant swarming species could always be isolated from a polymicrobial culture (such as 115 mammalian feces). In a competitive swarming assay, a mix of different pure bacterial cultures 116 gave rise to a single bacterial species populating the leading edge of the swarm colony on agar 117 (Fig. S1a-b). In congruence with our observation, a recent study has shown species dependence on 118 motility during niche dominance, and stable coexistence when present in low abundance in a mixed 119 population (Gude et al., 2020). Similarly, swarming assays using the pooled mouse or individual 120 human feces yielded single species of a dominant swarmer as identified by MALDI-TOF (STAR 121 Method Table 1; Fig. S1b). To test whether swarming bacteria are also present in preclinical 122 models, we screened feces of mice exposed to dextran sulfate sodium (DSS), a chemical colitogen 123 causing acute colonic inflammation (Chassaing et al., 2014a; Perse and Cerar, 2012). In a single 124 experiment, we found three identical isolates from two different mouse fecal specimens - Strain 1 125 from mice exposed to water and Strain 2 and 3 from mice exposed to dextran sulfate sodium (DSS), 126 respectively (Fig. S2a). Swarmers (in feces) were uniformly absent in water exposed mice, while 127 present in DSS exposed mice (Fig. 1e). We picked the edge of the swarm colonies (as marked on 128 Fig. S2a), then serially passaged twice on $1 \%$ agar from a single colony, and subsequently re-tested 129 for swarm behavior on $0.5 \%$ agar plates (Fig. S2b). Strain 3 swarmed significantly faster compared 130 to Strain 1 and 2. Interestingly, 16S rRNA gene analysis and Multi Locus Sequence Typing (Fig. 131 S2c) identified the isolated strains to be closest to Enterobacter asburiae. Whole-genome sequence 132 comparison of these Enterobacter strains (Fig. S2d) with related taxa Enterobacter asburiae and 133 Enterobacter cloacae, revealed that all the three strains isolated here were "nearly identical" 134 [>99\% identical, one contig of 5,107,194 bp (NCBI BioProject PRJNA558971)] and 135 phylogenetically distinct from the reference strains. Taken together, using an agar-based assay to 136 isolate dominant swarmers from a heterogenous culture, we were able to isolate nearly identical 137 strains with striking differences in their swarming potential. Strain 1 (Enterobacter sp. SM1) 
originated from feces of the vehicle (water) treated mice, while strain 2 (Enterobacter sp. SM2) and strain 3 (Enterobacter sp. SM3) originated from feces of DSS-induced colitis mice. Average Nucleotide Identity (ANI) analysis using OrthoANI (Yoon et al., 2017) found 96\% identity ANI between SM1/SM3 and E. asburiae. Interestingly, a quantitative PCR sequencing-based approach

142 to accurately identify SM1 or SM3 like bacteria in feces showed an increase in its abundance 143 during the evolution of DSS-induced colitis. The proportion of mice with high copy number values

$144(>10,000$ DNA copy number $/ \mu \mathrm{L})$ was significantly higher in the DSS group than water only group 145 (Fig. 1f).

146 Swarming Enterobacter sp. SM3 abrogates intestinal inflammation in a mouse model of colitis.

148 To determine the functional consequence of bacterial swarmers in the host, we administered the 149 "near-identical" swarming competent SM1 or SM3 strains to mice with DSS-induced colitis. In 150 comparison with SM1, SM3 is a hyperswarmer (Fig. S3a; Video S1), but both strains possess the 151 same swim speed (Fig. S3b, c), surfactant production (Fig. S3d) or growth rate (Fig. S3e). In 152 contrast to that observed with SM1, SM3 significantly protected mice from intestinal inflammation

153 (Fig. 2a-f). Comparison of clinical parameters showed that SM3 significantly protected from body 154 weight loss (Fig. 2a), increased colon length (Fig. 2b), reduced the colonic inflammation score 155 (Fig. 2d), and had reduced expression of pro-inflammatory mediators compared to vehicle-treated 156 colitic mice (Fig. 2e-f). To test the mucosal healing capacity of swarming bacteria, we 157 administered strains SM1 and SM3 to mice during the recovery phase of DSS exposure (Suzuki et 158 al., 2016). When compared to the vehicle, SM3 significantly improved weight gain and colon 159 length with reduced total inflammation and fibrosis at the microscopic level (Fig. 3). To identify 160 if the effect is flagella mediated, we used a TLR5KO IL-10R neutralization-induced colitis model 161 of mice. SM3 also significantly protected from body weight loss (Fig. 4a), reduced spleen and 162 colon weight (Fig. 4b-c), increased the cecum weight (Fig. 4d), reduced serum KC level and 163 lipocalin level (Fig. 4e-f), reduced levels of fecal lipocalin (Fig. 4g), reduced myeloperoxidase 164 activity (Fig. 4h), and had reduced the colonic inflammation score (Fig. 4i), when compared to the 165 SM1. We did not find differential regulation of any virulence associated genes between SM1 and 166 SM3 strains when collected from swarming plates (Fig. S3f-g). SM3 and its isogenic transposon 167 mutants (SM3_18 and SM3_24) only differed in swarming potential (Fig. S3h), but not swimming 
168

169

170

171

172

173

174

175

176

177

178

179

180

181

182

183

184

185

186

187

speed (Fig. S3i, j), surfactant production (Fig. S3k), or growth rate (Fig. S31). In mice exposed to DSS, SM3, but not the swarming deficient mutants (SM3_18 and SM3_24), showed significant protection against weight loss (Fig. 2g), colon length (Fig. 2h), and inflammation (Fig. 2i). During the course of experiment, on day 4, the levels of SM1, SM3 and its mutants present in feces were not significantly different (Fig. S4a-b). We chose to enumerate bacterial levels in feces on day 4 due to the equivalent pathological conditions of mice, as defined by weight change, when treated with different strains. To identify, if the loss of protection by SM3_18 could be related to slightly higher levels of its presence compared to SM3, although not significant, we performed a dose attenuation study. Even at low dose, the levels of inflammation as represented by lipocalin concentration and the weight loss was not significantly different from the vehicle group, negating the possibility of any associated virulence that may attribute to loss of protection by SM3_18 (Fig. S4e-f). Furthermore, we did not find significant difference of any virulence associated gene between SM3, and SM3_18, and SM3_24 strains when collected from swarming plates. Thus, in our mutants, pleiotropic effects of gene mutations on virulence is not a cause for lack of protection from inflammation. Together, however, these data indicated that SM3 with swarming properties, as opposed to swarming-deficient strains, is associated with anti-inflammatory activity. In accordance with these results, a diverse set of commensal swarmers (Bacillus subtilis 3610 and Serratia marcescens Db10) and a clinical strain of $S$. marcescens (isolated from the surface washing of a human dysplastic polyp) exhibited protection against DSS induced inflammation in mice (Supplementary Text and Fig. S5 \& S6).

\section{SM3 mediated abrogation of intestinal stress is microbiome dependent.}

To determine if the anti-inflammatory role of SM3 is dependent on the conventional intestinal microbiome composition, germ-free mice transferred to specific pathogen-free conditions (GF/SPF) and exposed to DSS-induced colitis, were treated with SM3. This strain was unable to abrogate intestinal inflammation in GF/SPF mice (Fig. 5a). We analyzed fecal samples of colitic mice (conventional and GF/SPF) with SM3 administered using 16S rRNA gene profiling. In contrast to GF/SPF mice, conventional mice feces showed specific enrichment of anaerobes belonging to the family S24-7 and Lactobacillaceae within SM3 treated mice when compared to vehicle mice (Fig. 5b). Specifically, in conventional mice, we found a significant increase in the abundance of S24-7 with SM3 gavage compared to vehicle in DSS exposed mice (Fig. 5c). 
However, quantitative PCR analysis of the levels of S24-7 in the feces of DSS-induced colitis mice gavaged with SM1 or SM3_18 or SM3_24, that did not exhibit protection from intestinal inflammation, was significantly reduced (Fig. 6a). In mice not exposed to DSS, the levels of S247 bacteria remain stable in SM3 treated group when compared with the untreated group (Fig. 5c).

202 Within DSS exposed conventional mice, we observed that enriched S24-7 negatively co-occurred 203 with pathogenic taxa such as the Peptostreptococcaceae and Enterobacteriaceae (Fig. 5d). 204 Together, these data suggest that protection from intestinal inflammation by SM3 is associated 205 with the presence of beneficial S24-7 group of bacteria (Volk et al., 2019).

\section{Enterobacter sp. SM3 promotes growth of Muribaculum intestinale in vitro.}

207 A recent study has reported the first cultured bacterium Muribaculum intestinale (DSM 28989) 208 that belongs to Bacteriodales S24-7 family (Lagkouvardos et al., 2019). We used this strain to 209 delineate any potential interspecies interaction with SM3 using an in vitro co-culture assay system.

210 However, in precedence, we assessed if the strain $M$. intestinale shared sequence homology to any 211 of the S24-7 taxa identified in our fecal 16S rDNA profile. OTU_5, which was found in highest 212 abundance among all other OTU's representing S24-7 taxa, exhibited $>96 \%$ identity to $M$. 213 intestinale (Fig. S7). Hence, we performed a broth-based co-culture assay using this strain and 214 SM3 or SM1 or SM3_18. Interestingly, the proportion of M. intestinale during co-culture was 215 higher compared to its monoculture at any tested time point. SM3 as well as the partially swarming 216 deficient strains, SM1 and SM3_18, had a two-four (2-4) fold increase in DNA copy number/ $\mu \mathrm{L}$, 217 when analyzed by qPCR using S24-7 specific primers (Fig. 6b). We also designed and developed 218 a plate-based co-culture assay to compare the effects of swarming bacteria SM3 and swarming219 deficient variants, SM1 or SM3_18, on the growth of M. intestinale. In this assay, swarming plates 220 harbored a central bore well containing $M$. intestinale that guarantees a direct or indirect interaction 221 with the spreading bacteria on agar of the same plate. The plates were sealed so that the act of 222 swarming generated an anaerobic environment suitable for the growth of M. intestinale. At 64 223 hours, in congruence with the broth co-culture assay results, we observed an increase in $M$. 224 intestinale counts with SM3, SM3_18, and SM1 (Fig. 6c). To better understand if the observed 225 increase in $M$. intestinale levels is mediated by a direct or an indirect interaction during the co226 culture studies, and not solely due to the reduced oxygen concentration in the agar plate, we 227 developed a separate plate-based co-culture assay. In this assay, the swarming region was 
physically separated from the central bore well containing $M$. intestinale to prevent any direct or indirect interaction with the swarming bacterium. In this system, as the bacteria swarmed on the agar surface over $64 \mathrm{~h}$, oxygen levels were reduced. $M$. intestinale showed no growth under the conditions tested (Fig. 6c, Divided/Sealed). Overall, our results suggest, that both planktonic and

232 swarming cells of SM3, SM1, or SM3_18, when co-cultured in vitro, can promote the growth of

233 S24-7 family (M. intestinale), independent of reduced oxygen concentrations in the environment.

234 Coincidently, the development of significantly reduced oxygen concentrations in the environment

235 are also observed in vivo but only with SM3 and not SM1 or the other SM3 mutant bacteria

236 (Supplementary text and Fig. S8). Our results suggest that SM3 proximity to M. intestinale is

237 necessary for the induction of the growth of the latter species.

238 The act of bacterial swarming is likely a phenomenon in vivo.

239 The intestinal mucosa is relatively uneven during inflammation due to the loss of mucin (Fig.

240 S10)(Sasaki et al., 2008). We conjectured, therefore, that swarmers might have an added 241 advantage in niche dominance on inflamed tissue. Indeed, a race assay designed on mouse mucosal 242 surface demonstrated the biological relevance of swarming. On mouse mucosa, it is not possible 243 to visualize bacterial cells directly, so we developed the mucosa racing experiment to determine 244 the dominant motility type. We developed a hyperswarmer strain of SM1, HS2B, that swims 245 slower but swarms at a similar rate and extent to that of SM3, and an isogenic mutant $\Delta f l h E$ SM1 246 that is swarm deficient but swim competent when compared to SM1. In this experiment, identically 247 sized segments of "normal or control" mouse colon was placed on a hybrid agar plate $(1 \% / 0.3 \%)$ 248 (see STAR Method) and equal concentration (CFU/mL) of bacteria spotted. As a negative control, 249 sterile LB and a non-motile strain of $\Delta$ motA SM1 when spotted did not show any motility across 250 the mucosal surface even after 20h (Video S4). In comparing the "motility rates" across the normal 251 colon mucosa (see STAR Method), the hyperswarming HS2B demonstrated slow motility when 252 compared to $\Delta f l h E$ SM1 (Fig. S11c). However, when the same experiment was performed using 253 inflamed mouse colon after exposure to DSS in vivo, the motility of HS2B was significantly faster 254 than swarming deficient $\Delta$ flhE SM1 (Fig. S11d). We also used S. marcescens strains, swarming 255 Db10 and non-swarming JESM267 ( swrA) mutant to confirm our hypothesis. While on normal 256 colon mucosa Db10 and JESM267 did not significantly differ in the motility rates, however, Db10 257 was significantly faster than JESM267 on colitic mucosa (Fig. S11g-h). This indicates that 
swarming bacteria finds an advantage in motility on a colitic mucosa compared to normal mucosa

259 (Video S2-4). Parenthetically, we have recently developed and used a distinct

260 Polydimethylsiloxane (PDMS) based confinement tool to show that a unique motility pattern

261 associated with bacterial swarming is also seen on tissue from colitic mice gavaged with SM3 (doi:

262 https://doi.org/10.1101/2020.08.30.274316). Furthermore, to understand if SM3 does swarm in

263 vivo, we searched for the presence of transcriptomic markers in the feces that can be linked to SM3

264 swarming physiology. In agar-based studies of RNA sequencing of SM3 obtained from the edge

265 of a swarming colony versus the pre-swarming colony at center, a singular pathway was

266 significantly upregulated - the lipid A biosynthetic pathway (fold change 3 fold, q value $=0.0376$ ).

267 Meta-transcriptomic analysis of feces from SM3 treated DSS induced colitic mice identified steady

268 increase of lauroyl acyltransferase transcript involved in Lipid A biosynthesis on Day 4 and Day

26912 when compared to Day 0 (Fig. S12a). However, heat killed SM3 treatment showed reduction

270 in transcript abundance by Day 12. Other genes known to be associated with swarming such as the

271 sigma factor FliA and nitrate reductase NarH were also enriched in SM3 versus heat killed SM3

272 gavaged colitic mice (Fig. S12b). Normal mice gavaged with SM3 or heat killed SM3 did not show

273 enrichment of these genes. Collectively, our data provides multiple lines of evidence suggesting

274 that bacterial swarming is a likely phenomenon in vivo, and a motility form that is necessary for

275 the induction of $M$. intestinale growth.

276 Discussion

277 Our study finds that intestinal inflammation itself promotes a protective niche that allow

278 enrichment of bacterial swarmers. The inflammatory milieu likely provides a permissive

279 environment for stress adaptation and swarming behavior. Surprisingly, however, these bacterial

280 swarmers when dosed in sufficient abundance abrogate intestinal inflammation in mice. We

281 focused on a novel bacterium, Enterobacter sp. SM3, which is resident to the intestinal microflora

282 of mice. In vivo, SM3, but not SM1, or SM3 swarming deficient mutants (poor swarmers),

283 influenced the specific enrichment of S24-7 group of bacteria. Notably, the family of S24-7

284 (Muribaculaceae) are known to repair barrier function in inflamed mice intestines (Osaka et al.,

285 2017; Volk et al., 2019). However, the in vitro co-culture experiment proved that a close

286 interaction between SM3 and S24-7 group of bacteria is essential for its enrichment. Thus, we

287 hypothesized that it is the relative hyperswarming activity of SM3 (but not very slow swarming 
SM1 or SM3 mutants) that may facilitate a close interaction with S24-7 group of bacteria, in vivo.

289 Further support of this hypothesis comes from the ability of bacteria to swarm on a mucosal surface afflicted only by colitis (ex vivo mucosal race assay) and the meta-transcriptomic analysis demonstrating that at least two major metabolic pathways known to be associated with motility are upregulated in coltic mice administered SM3 (but not its heat-killed counterpart). The present mechanism implicates swarming SM3 to directly enhance S24-7 (Muribaculaceae) which then suppresses host inflammation (Graphical Abstract). Nevertheless, we do not exclude other direct or indirect effects of the swarming SM3 on mucosal inflammation and healing. However, if present, it would assist in suppressing host inflammation in conjunction with enrichment of S24-7 group of bacteria in the gut.

Increased prevalence of swarmers from stressed intestinal contents of mammals (humans, pigs, and rodents) encouraged us to investigate its relevance on host health during colitis. We developed a method to isolate dominant swarmers resident in the host from fecal and colonoscopic washing samples. Screening of several bacterial species allowed us to identify genetically identical gramnegative bacterial strains SM1 and SM3 from feces of normal and DSS induced colitic mice, respectively. Bacterial size (Be'er et al., 2013), swimming speed (Sokolov and Aranson, 2009), cell density (Zhang et al., 2010), and several other factors (Angelini et al., 2009) are known to affect swarming and swarm patterns in vitro. Comparing these factors that could affect the swarming ability of SM1 and SM3, we found that the swimming speeds and growth rates were similar (Fig. S3a-e). Swarming bacteria secrete surfactants, such as surfactin, that facilitate during motility on a solid surface (Kearns, 2010). As surfactin is reported to attenuate TNBS induced colitis, possibly by differentially regulating anti-inflammatory and pro-inflammatory cytokines (Selvam et al., 2009), we determined surfactant levels using blood agar assay, drop-collapse assay (Bodour and Miller-Maier, 1998) and drop-counting assay based on modified Stalagmometric

312 Method (Dilmohamud et al., 2005). None of the strains showed significant difference in surfactant 313 production when compared to its isogenic mutants, at the conditions tested (Supplementary text, 314 Fig. S13).

315 Furthermore, as DSS is a polymer of sulfated anhydroglucose we performed a separate experiment 316 to show that SM3 does not influence DSS induced cell cytotoxicity using a cell line model of 317 intestinal epithelial differentiation (Caco-2) (Fig. S14). Thus, it is unlikely that SM3 can either 
metabolize and/or inactivate DSS per se. To identify if the pathophysiological effect observed with bacterial swarmers was specifically due to its swarming physiology, we focused on generating isogenic mutants that were swarm deficient but proficient in all other features that may otherwise affect swarming (swimming, growth rate, surfactant production). To emphasize the importance of

322 functional flagella in protection during intestinal inflammation, we used a motor protein abrogated strain of SM1 ( $\Delta$ motA SM1) in mice with DSS induced colitis. Notably, this mutant strain that

324 had equivalent growth rate and an intact flagellum but was unable to either swim or swarm showed 325 no protection against intestinal inflammation in a DSS mice model study (Fig. S15). As a proof of concept that swarming SM3 protects from inflammation, we used two isogenic strains SM3_18

327 and SM3_24 that had significantly reduced potential to swarm, albeit with similar swimming 328 speeds and growth rates compared to the wildtype. Transpositions in SM3_18 and SM3_24 were 329 found to locate within the putative structural genes encoding N6-hydroxylysine O330 acetyltransferase or aerobactin synthesis protein (iucB) and isocitrate/isopropylmalate dehydrogenase/ADP-ribose pyrophosphate of COG1058 family, respectively. Nevertheless, transposon integration in SM3_18 led to a polar insertion that will only disrupt the expression of

333 downstream genes $i u c C$, iucD and $i u t A$ located within the operon, hampering aerobactin synthesis 334 only. Fundamentally, genes $i u c D$ and $i u t A$ aid in iron acquisition in bacteria during nutrient 335 limiting condition (Koster et al., 1994). A single study has also shown the dependence of bacterial 336 phytopathogen Pantoea stewartii swarming on aerobactin synthesis (Burbank et al., 2015). 337 Similarly, in SM3_24 non-polar insertion within the gene coding for putative 338 isocitrate/isopropylmalate dehydrogenase/ADP-ribose pyrophosphate of COG1058 family, may 339 not affect the transcription of downstream gene that has two possible promoter sequences at the 340 3'end of the insertion site, as determined by BPROM (Solovyev, 2011).

341 TLR-5 knockout mice fail to express anti-flagellin antibodies, an immunomodulatory pathway that 342 aids in maintaining intestinal homeostasis (Cullender et al., 2013). Even in the absence of TLR5, 343 colitic mice harboring normal microbiota showed significant protection when treated with SM3. 344 In contrast, in a germ-free condition, SM3 lost protection allowing us to speculate the role of 345 intestinal microbiome in the observed effect. Indeed, oral gavage of SM3 in conventional colitic 346 mice showed enrichment of beneficial anaerobes and microaerophiles. These anaerobes belong to 347 the family Bacteroidales S24-7 and Lactobacillaceae. S24-7, recently classified to Muribaculaceae 348 family, forms one of the major taxa in mouse gut (Lagkouvardos et al., 2019) and has been 
associated with disease remission (Borton et al., 2017; Osaka et al., 2017). Similarly, Lactobacillus producing lactate is known to promote the proliferation of intestinal epithelial cells (Okada et al., 2013). In view of the fact that oxygen content of the intestinal lumen increases during intestinal inflammation (a shift from anoxic to oxic)(Colgan and Taylor, 2010) (Fig. S8a), it was unexpected to find enrichment of obligate anaerobes such as Bacteriodales S24-7 in SM3 treated mice. Recent metagenomic analyses have revealed the potential of S24-7 bacteria to be "nanaerobe", permitting growth in nanomolar concentrations of oxygen (Ormerod et al., 2016). This observation allowed us to hypothesize the possible role of SM3 in the rapid depletion of oxygen in the lumen, and in turn, favoring the growth of resident anaerobes. In congruence, we observed SM3 fed colitic mice had significantly lower oxygen concentration compared to the colitic mice treated with swarming deficient variants. We conjectured the possible role of swarming movement of SM3, if occurring in vivo, in reducing oxygen concentration. To link swarming activity and oxygen necessity, we showed by in vitro experiments that SM3 cannot swarm at low concentrations of oxygen. It was further corroborated by the increase in anaerobic taxa in the feces of GF/SPF mice treated with SM3. Nevertheless, a steady increase of S24-7 specific OTU's in SM3 treated DSS-colitic mice pointed towards a potential mechanism underlying the observed protection. Hence, we designed a broth and plate-based co-culture assay to identify possible specific interaction between SM3 and the first cultured bacterium belonging to the S24-7 family, M. intestinale. Both SM3 and the less swarming variants promoted growth of $M$. intestinale in co-culture assay. However, linking this observation with decrease in the levels of S24-7 in the fecal DNA obtained from SM1, SM3_18, and SM3_24 led us to speculate the essential role of swarming by SM3 in exhibiting protection. We conjectured that in addition to an anaerobic environment generated by the act of swarming on the agar plate, all the tested strains either required a direct cell-cell contact or produced a 372 secretome, which promoted growth of $M$. intestinale. This was further validated by a plate-based 373 assay that allowed physical separation of swarming SM3 from $M$. intestinale, but at the same time 374 creating an anaerobic condition in the system suitable for the growth of M. intestinale. Together 375 this suggest, a close spatial interaction between SM3 and S24-7 group of bacteria allow enrichment of the latter preferably in a microenvironment. In vivo, SM3 may aid in re-establishing hypoxia, 377 and consequently creating an optimal environment for the growth of S24-7 and other anaerobes. 378 The present mechanism strongly suggests that swarming (but not swimming or slow swarming) 
SM3 directly enhances S24-7 (Muribaculaceae) by attaining proximity to it in vivo (Graphical Abstract).

In broadening the scope of our hypothesis, we observed that even known commensal swarming strains Bacillus subtilis 3610 and Serratia marcescens Db10 protected against DSS-induced colitis in mice when compared to its isogenic swarming deficient mutants. Similar results were also observed with a clinical strain of $S$. marcescens isolated from the colonoscopic aspirates of a human colitis patient, which also swarmed in vitro. Since this Serratia isolate was obtained from human feces and not characterized at the genome sequence level, we used wildtype and heat-killed clinical strains to study their effects on mice with DSS-induced colitis. We found that the wildtype, but not the heat-killed strain protected against colitis (Fig. S6). Such protection observed with different bacteria belonging to a distinct phylogenetic group may also preclude the possibility of any loss of protection seen in case of isogenic mutants due to other pleiotropic effects. Similarly, a pathogenic swarmer strain of Salmonella enterica serovar Typhimurium had reduced severity and less inflammation compared to its swarm defective mutant $\Delta f l i \mathrm{~L}$ (Fig. S16, Supplementary text). Overall, these observations suggested that bacterial swarmers and likely the act of swarming play a major role in mitigating disease severity in a mouse model of colitis.

Bacterial swarming is a fundamental process in certain groups of bacteria characterized by collective and rapid movement across a surface (Kearns, 2010), (Be'er and Ariel, 2019) and has been reported in vitro. We focused on understanding if swarming is likely a phenomenon in vivo. As an initial approach, we developed an ex vivo mucosal race assay that proved motility dominance of bacterial swarmers on a biological surface. Similarly, we have developed a PDMS based tool to 400 distinguish bacterial swimming from swarming, which exhibited the possibility of the latter movement in micro pockets on DSS treated mucosal tissue. However, technical limitations to 402 demonstrate swarming in vivo in a mouse model limits generalization of our data. First, in vivo, 403 intestines are continually moving through a process termed by Baylis \& Starling as "peristalsis" 404 (Spencer et al., 2016). These myogenic ripples actively move food and other particles along the 405 intestines to the anal region for defecation. If we could image our bacteria in vivo, the artifact of 406 peristalsis would cloud any interpretation of swarming movement in vivo. Further, poor resolution 407 in contemporary microscopic endoscopy is a limitation. Second, in order to detect collective 408 motion in vivo, we would need to image the bacteria studied. GFP labeling of SM3 knocks out its 
swarming ability. We have also labeled the outer membrane of SM3 using click chemistry,

410 however, upon gavage, we posit that the signal will be lost as the bacteria replicate in the

411 gastrointestinal tract. Both limitations, at present, preclude any ability to efficiently and

412 functionally label SM3 in vivo. Due to these limitations, we have demonstrated the possibility of

413 swarming movement in vivo by identifying genes that are known to be associated with swarming.

414 One of the upregulated genes in SM3 treated colitic mice, Lauroyl acyltransferase, is an enzyme

415 involved in lipopolysaccharide (LPS) biosynthesis, which forms an integral part of cell envelope

416 biogenesis. LPS synthesis genes are upregulated in swarmer cells that act as a lubricant, promoting

417 swarming (Partridge and Harshey, 2013). Arguably, LPS is an important biomarker during

418 intestinal inflammation (Im et al., 2012). Although KEGG pathway analysis showed enrichment

419 of LPS biosynthesis pathways in SM3 treated colitic mice compared to Heat killed SM3 treatment,

420 protection observed may indicate the upregulated pathways in association with bacterial swarming.

421 The nitrate reductase beta chain, $\mathrm{NarH}$, involved in nitrogen dissimilation is also linked to

422 rhamnolipid biosynthesis in P. aeruginosa (Van Alst et al., 2007). Rhamnolipids are essential for

423 swarming behavior in Pseudomonas (Caiazza et al., 2005). A similar role for NarH could be at

424 play in Enterobacter sp. SM3, as there is a significant increase in nitrate reductase beta-chain

425 transcripts found in pre-swarming SM3 cells collected from the center of the agar plate $(\mathrm{q}=1.27 \mathrm{E}-$

426 224). Two-component system analysis using fecal metatranscriptome also identified pathways

427 with upregulation of specific sigma factors. FliA is an alternate sigma factor that directs

428 transcription of Class III flagellar genes and is also essential in bacterial swarming(Young et al.,

429 1999). The HptB-sigma factor signaling system, as identified in KEGG analysis (Fig S12b), also

430 utilizes FliA to upregulate flagellar biogenesis essential in swarming by $P$. aeruginosa(Bhuwan et

431 al., 2012). Overall, these data together support the notion that swarming movement likely occurs

432 in vivo.

433 In summary, our work demonstrates the unique and unprecedented role that bacterial swarmers 434 play in intestinal homeostasis. Our work demonstrates the potential for a new personalized 435 "probiotic" approach stemming from the ability to isolate and bank swarming microbes during 436 colitis flares for clinical treatment during concurrent or subsequent colitic episodes. 


\section{Acknowledgement}

439 We thank Steve Almo, Andrew Gewirtz, Cait Costello, Jeffrey Pessin, Matthew R. Redinbo and

440 John March for valuable discussions. We aslo thank Brad Tricomi for developing the assay

441 "Cytotoxicity of DSS on Caco-2 cell lines in the presence or absence of viable SM3 cells", Ehsan

442 Khafipour for providing pig specimens (feces) and performing clinical scoring of histopathology,

443 Cori Bargmann at Rockfeller University for gifting us the bacterial strains Serratia marcescens

444 Db10 and JESM267. Additional assistance was obtained from Amanda Beck DVM (Histology and

445 Comparative Pathology Core, AECOM), Olga C. Arionadis, Thomas Ullmann and Azal Al Ani

446 (Department of Medicine, AECOM), Winfred Edelmann and Eleni Tosti (Department of Cell

447 Biology, AECOM). The studies presented here were supported in part by the Broad Medical

448 Research Program at CCFA (Crohn's \& Colitis Foundation of America; Grant\# 362520) (to S.M);

449 NIH R01 CA127231; CA 161879; 1R01ES030197-01 and Department of Defense Partnering PI

450 (W81XWH-17-1-0479; PR160167) (S.M.), Diabetes Research Center Grant (P30 DK020541);

451 Cancer Center Grant (P30CA013330 PI: David Goldman); 1S10OD019961-01 NIH Instrument

452 Award (PI: John Condeelis); LTQ Orbitrap Velos Mass Spectrometer System (1S10RR029398);

453 and NIH CTSA (1 UL1 TR001073). Peer Reviewed Cancer Research Program Career

454 Development Award from the United States Department of Defense (CA171019, PI: Libusha 455 Kelly). 


\section{Author Contributions}

458 H.L., S.M. conceptualized the discovery. H.L., D.K., W.C., J.T., S.M. designed and executed the 459 swarming assays. D.L. was the Principal Investigator of the Clinical Study and provided 460 specimens. L.K. performed genome assembly and annotation. J.W., R.L., S.M. designed and 461 executed all the 16S, metagenomic and strain-specific PCR assays. A.D. designed; A.D., W.C., 462 S.M., S.G. characterized bacterial mutants. B.S.Y. and M.V-K. performed tlr5KO mice study and 463 repeat swarming assays for reproducibility. A.D., H.L., W.C. and S.M. wrote and edited the paper. 464 S.C. and W.C. performed statistical analyses. X.L. assisted H.L. in mouse model studies. S.G. has 465 performed a single independent mice model study. A.B. analyzed the clinical data and revised the 466 paper. K.S. did the histological preparations and examination. C.J. and Z.H. performed gnotobiotic 467 mouse model studies. W.S. identified bacteria strains using MALDI-TOF.

\section{Declaration of Interests}

469 Sridhar Mani, Libusha Kelly, and Hao Li filed a U.S. patent application (Application No. 470 62237657). Other authors declare no competing financial interests. 
bioRxiv preprint doi: https://doi.org/10.1101/759886; this version posted October 7, 2020. The copyright holder for this preprint (which was not certified by peer review) is the author/funder, who has granted bioRxiv a license to display the preprint in perpetuity. It is made available under aCC-BY-NC-ND 4.0 International license.

\section{Figure 1}

a

\begin{tabular}{cccc}
\hline \multirow{2}{*}{ Age } & & \multicolumn{2}{c}{ Disease $^{*}$} \\
\cline { 3 - 4 } & Mean ( \pm SD) & $51 \pm 14$ & $50 \pm 11$ \\
\multirow{2}{*}{ Gender } & Median (Range) & $52(21-81)$ & $51(24-70)$ \\
& Females & 27 & 8 \\
& Males & 17 & 9 \\
\hline
\end{tabular}

* Defined as individuals with clinically established active inflammatory bowel disease (Crohn's disease $n=14$, or Ulcerative colitis $n=5)$, intestinal dysplasia $(n=1)$, intestinal polyps $(n=23)$, intestinal reactive hyperplasia and inflammatory changes not otherwise specified $(n=1)$. All other diagnoses were considered "negative" for disease.

C

\begin{tabular}{ccc}
\hline Statistic $^{*}$ & Value & $95 \% \mathrm{Cl}^{\dagger}$ \\
\hline Sensitivity & $55.56 \%$ & $40.00 \%$ to $70.36 \%$ \\
Specificity & $88.00 \%$ & $68.78 \%$ to $97.45 \%$ \\
Positive Likelihood Ratio & 4.63 & 1.55 to 13.81 \\
Negative Likelihood Ratio & 0.51 & 0.35 to 0.72 \\
Disease Prevalence & $64.29 \%$ & $51.93 \%$ to $75.39 \%$ \\
Positive Predictive Value & $89.29 \%$ & $73.63 \%$ to $96.13 \%$ \\
Negative Predictive Value & $52.38 \%$ & $54.88 \%$ to $77.91 \%$ \\
\hline
\end{tabular}

"Computed with: https://www.medcalc.org/calc/diagnostic_test.php

${ }^{\dagger} \mathrm{Cl}$, confidence interval

e
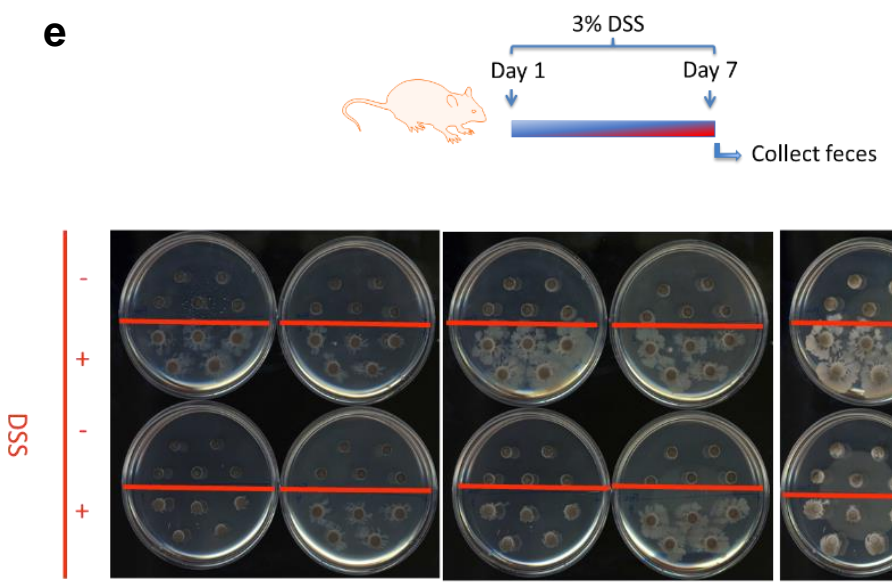

$\mathrm{t}=12 \mathrm{~h}$

$\mathrm{t}=24 \mathrm{~h}$

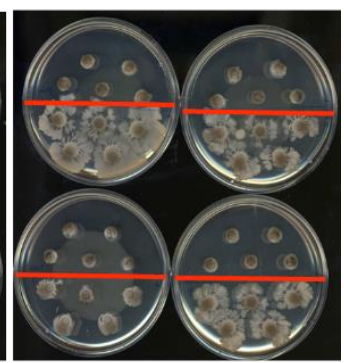

$\mathrm{t}=72 \mathrm{~h}$ b

\begin{tabular}{lccc}
\hline & & \multicolumn{2}{c}{ Disease $^{*}$} \\
\cline { 3 - 4 } & & + & - \\
\hline \multirow{2}{*}{ Swarming test $\dagger$} & Swarming & 25 & 3 \\
& Non-swarming & 20 & 22 \\
\hline
\end{tabular}

* One patient consented for the study specimen but did not undergo colonoscopy; One patient had a poor colonoscopy preparation and aspirate sample was not obtained; 9 fecal samples obtained from OpenBiome (Boston, MA), one of which was contaminated inadvertently with a laboratory swarming strain and was excluded from analysis.

† "Swarming" defined as swarming score $\geq 1$; "Nonswarı " defined as swarming score $=0$.
Figure 1 | Effect of intestinal inflammation on bacterial swarming. a-c, Human colonoscopy aspirates $(\mathrm{n}=45$ intestinal disease; $\mathrm{n}=25$ non-disease) were spotted on $0.5 \%$ agar plates and the swarming assay performed. $\mathbf{a}_{2}$ Colonoscopic washes were obtained from individuals with active intestinal disease and matched controls. Swarming assays performed using aspirates were binned by disease as defined both clinically and by intestinal histopathology, where available. b, Clinical demographics are described for the disease and non-disease population. c, Swarming assays' clinical test characteristics. d, Swarming assays (72h) of fecal samples collected from pigs with and without IBD. Swarming scores - 0: no swarming, 1: swarming within 72h, 2: swarming within 48h, 3: swarming within 24h or less (Control: $\mathrm{n}=6$; IBD: $n=7$, each in triplicate, sampled from distinct regions of the semi-solid feces). e, C57BL/6 mice (8-week old) were exposed to water or DSS water for 7 days $(n=4$ per group). Fecal samples of control group (above red line) and DSS group (below red line) were collected for swarming assay. Swarming plates were scanned at 12, 24 and 48 hours. f, In a separate experiment, C57BL/6 mice (8-week old) were exposed with water or DSS water for 12 days ( $n=8$ per group). Fecal samples were collected for DNA extraction and SM1/SM3-specific PCR analysis was performed, and DNA copy number ascertained. Unless otherwise noted, data represented as mean and 95\% CI, significance tested using Fisher's Exact test. 
bioRxiv preprint doi: https://doi.org/10.1101/759886; this version posted October 7, 2020. The copyright holder for this preprint (which was not certified by peer review) is the author/funder, who has granted bioRxiv a license to display the preprint in perpetuity. It is made available under aCC-BY-NC-ND 4.0 International license.

Figure 2

a

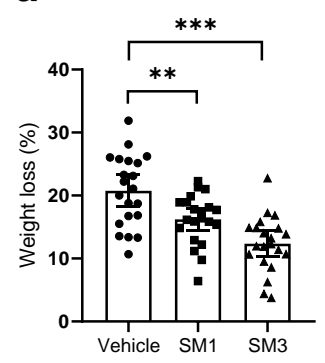

b

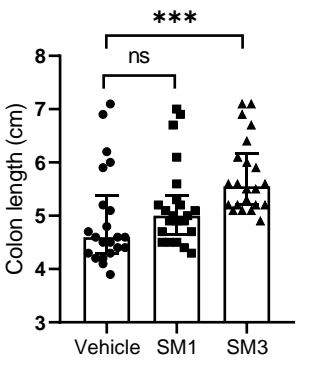

C

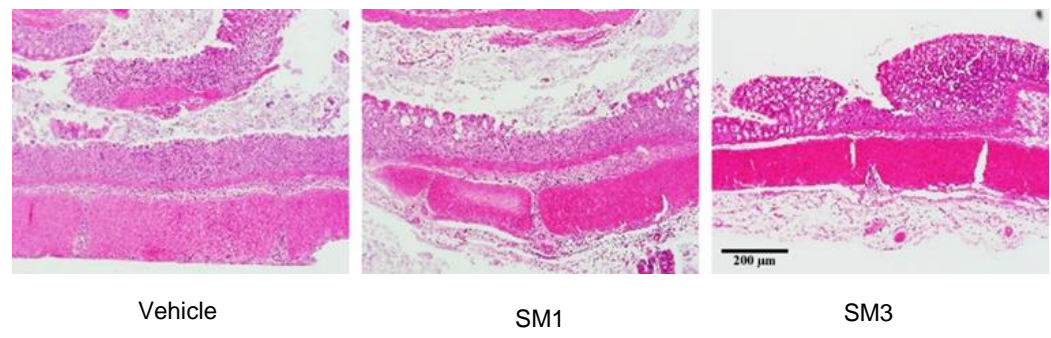

d

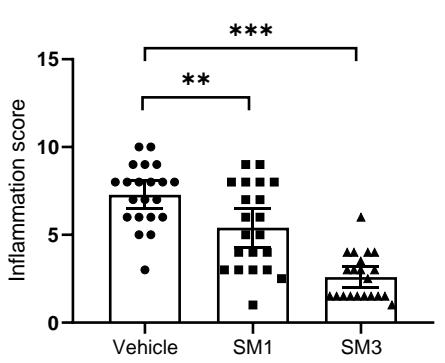

g

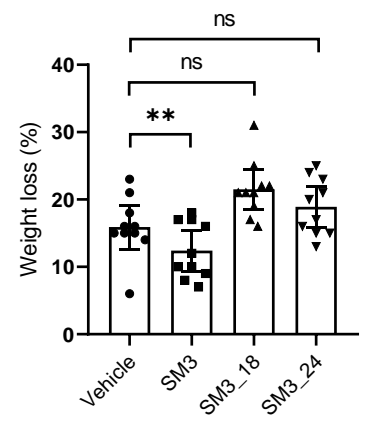

e

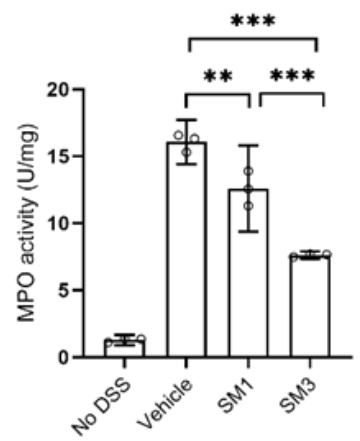

h f

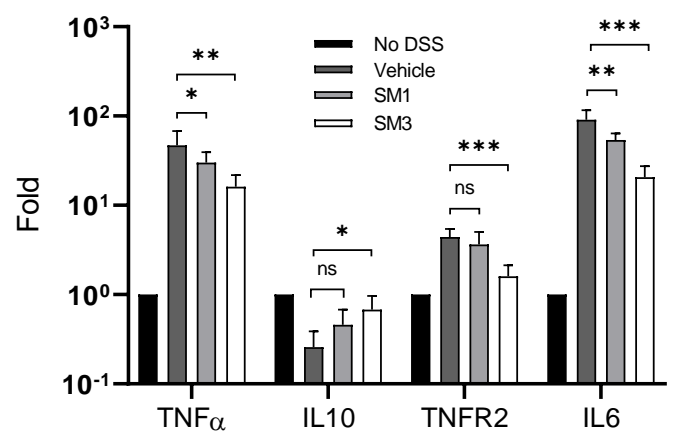

i

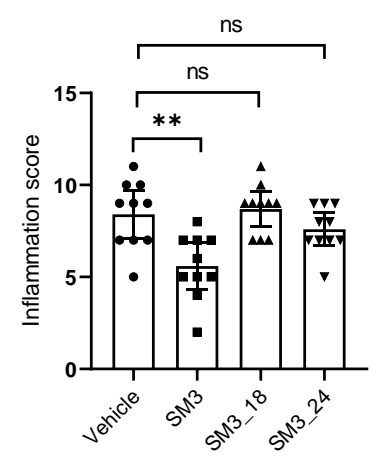

Figure $2 \mid$ Effects of Enterobacter sp. SM strains on DSS induced colitis in C57BL/6 mice. a-f, 8-week old mice were exposed to DSS water and treated with vehicle (LB), SM1 or SM3 by oral gavage for 10 days. a-b indicates weight loss (a) and colon length $(\mathbf{b})(\mathrm{n}=21$ per treatment group). c, Representative images (100x magnification) of H\&E stained colonic section treated with vehicle (left), SM1 (middle) and SM3 (Borton et al.). d, Inflammation score ( $\mathrm{n}=21$ per treatment group). e-f, In a separate experiment, myeloperoxidase (MPO) enzyme activity was determined $(n=3$, each in duplicate) $(\mathbf{e})$. Colon total RNA $(n=4)$ was isolated and reverse transcribed to cDNA. RT-qPCR data show fold induction of mRNA (TNF $\alpha$, IL10, TNFR2, IL6). PCR was repeated in quadruplicate. The expression was normalized to internal control, TBP. The entire experiment was repeated $n=2$ for reproducibility (f).g-i, In a separate experiment, C57BL/6 mice (8-week old) were exposed to DSS water and administered vehicle (LB), SM3, or its mutants (SM3_18 or SM3_24) for 10 days. g-i indicates weight loss (g), colon length (h) and inflammation score (i) $(\mathrm{n}=10$ per treatment group). Unless otherwise noted, data are represented as mean and $95 \% \mathrm{CI}$, and significance tested using one-way ANOVA followed by Tukey's post hoc test. c, data represented as median and interquartile range, and significance tested using Kruskal-Wallis followed by Dunn's multiple comparisons test. * $P<0.05$; ** $P<0.01$; *** $P<0.001$; ns, not significant. H\&E, Hematoxylin and Eosin; TBP, TATA-Box Binding Protein; CI, Confidence Interval. 
bioRxiv preprint doi: https://doi.org/10.1101/759886; this version posted October 7, 2020. The copyright holder for this preprint (which was not certified by peer review) is the author/funder, who has granted bioRxiv a license to display the preprint in perpetuity. It is made available under aCC-BY-NC-ND 4.0 International license.

\section{Figure 3}

a

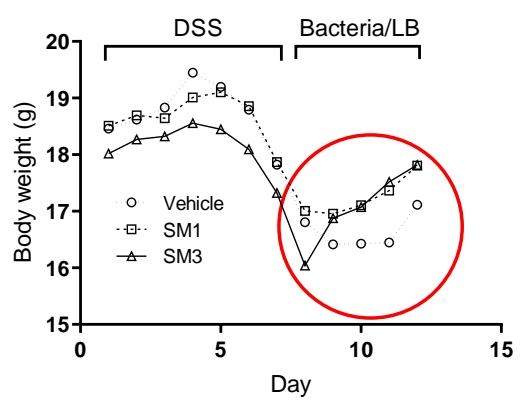

d

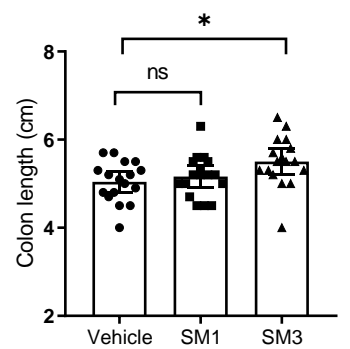

b

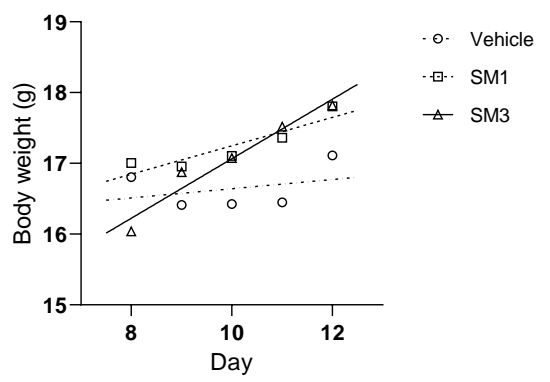

e

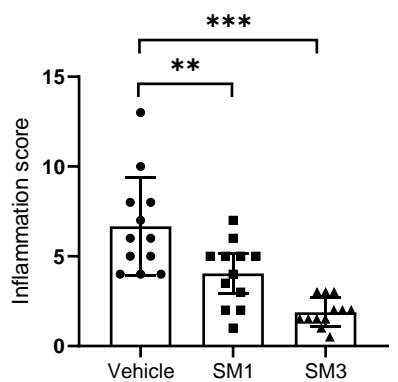

C

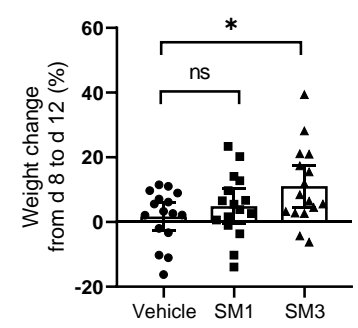

f

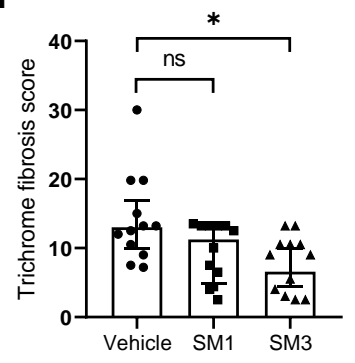

Figure 3 Effect of Enterobacter sp. SM1 or SM3 strain on DSS induced colitis in C57BL/6 mice during recovery phase. 8-week old mice were exposed to DSS water for 7 days. On day 8, DSS water was replaced with drinking water and mice were administered vehicle (LB), SM3 or SM1 for 5 days. a-c, indicates the weight change. a, Day by day weight change. b, Day by day weight change from day 8 to day 12 (healing phase, red circle in a) was separately plotted, and the best fitting line was added to each group using linear regression. The slopes for the regression lines are 0.421 (SM3), 0.201 (SM1) and 0.065 (Vehicle). The slope of SM3 group is significantly deviated from zero (P = $0.013)$, while the SM1 and vehicle group are not $(P=0.240,0.754$ respectively). c, Percent weight change from day 8 to day 12 . d-f, indicates colon length (d), inflammation score (e), and trichrome fibrosis score (f) $(n=16$ per treatment group except for $\mathbf{e}$ and $\mathbf{f}$, four colon specimens per group were used for other experiments). Unless otherwise noted, data represented as mean and $95 \% \mathrm{CI}$, and significance tested using oneway ANOVA followed by Tukey's post hoc test. f, data represented as median and interquartile range, and significance tested using KruskalWallis followed by Dunn's multiple comparisons test. 
bioRxiv preprint doi: https://doi.org/10.1101/759886; this version posted October 7, 2020. The copyright holder for this preprint (which was not

473

474

475

\section{Figure 4}

a

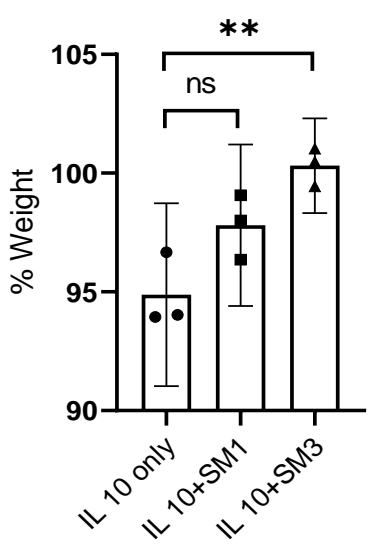

d

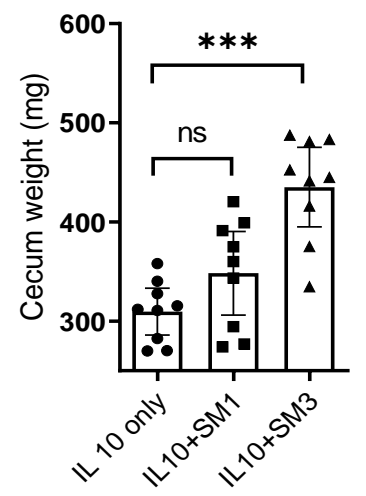

g

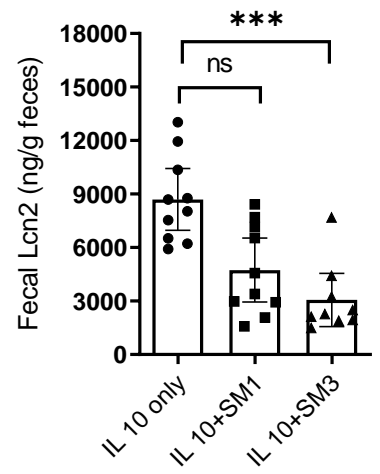

b
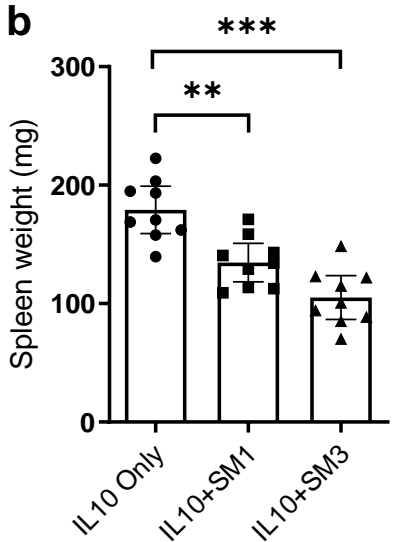

e
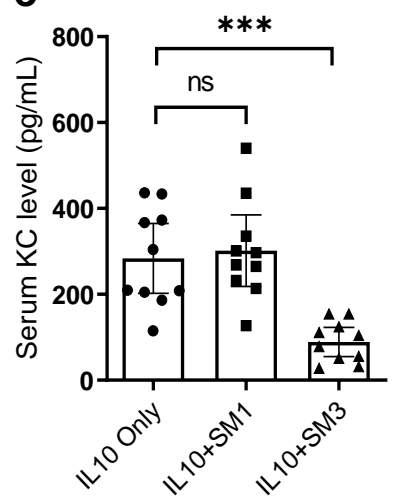

h

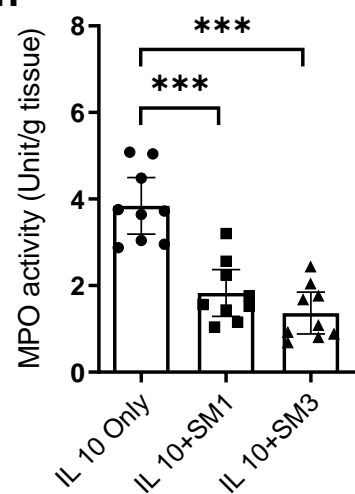

C

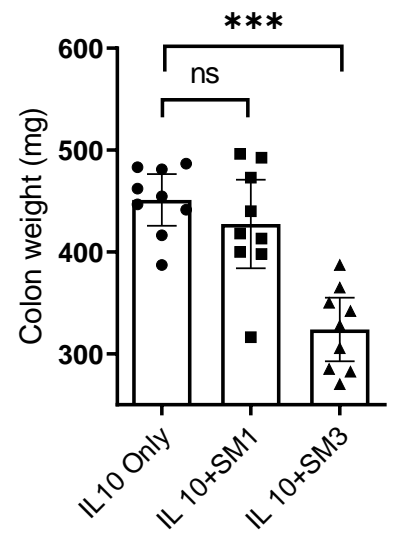

f

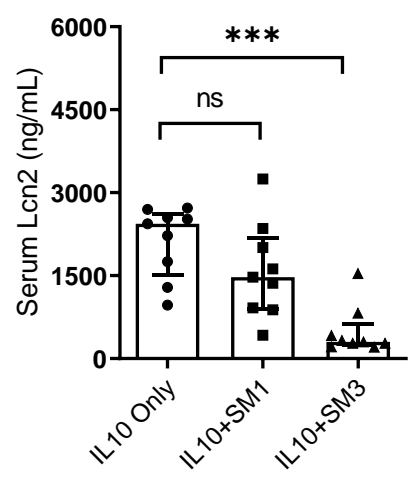

i

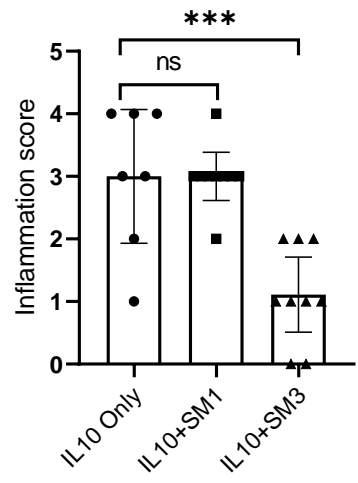

Figure 4 | Effects of Enterobacter sp. SM strains on IL-10R neutralization-induced colitis in Tlr5KO mice. 8-week-old Tlr5KO mice were administered rat anti-IL-10R monoclonal antibody (1.0 mg/mouse, i.p.) (BioXcell) at day 0 and 7. SM1 or SM3 was gavaged every $3^{\text {rd }}$ day from day 1 until day 18. a, Weight percentage compared with day $0(n=3$ per treatment group). b, Spleen weight $(n=9$ per treatment group). c, Colon weight $(n=9$ per treatment group). d, Cecum Weight $(n=9$ per treatment group). e, Serum KC level $(n=10$ per treatment group). f, Serum lipocalin ( $\mathrm{n}=9$ per treatment group). $\mathbf{g}$, Fecal lipocalin $(\mathrm{n}=10$ for each group, 1 fecal sample in SM3 group was used for other study). h, Myeloperoxidase (MPO) $(n=9$ per treatment group). i, Inflammation score $(n=9$ for each group, 2 tissue samples in IL 10 only group were used for other study). Unless otherwise noted, data are represented as mean and 95\% CI, and significance tested using one-way ANOVA followed by Tukey's post hoc test. f-g, data represented as median and interquartile range, and significance tested using Kruskal-Wallis followed by Dunn's multiple comparisons test. 
bioRxiv preprint doi: https://doi.org/10.1101/759886; this version posted October 7, 2020. The copyright holder for this preprint (which was not certified by peer review) is the author/funder, who has granted bioRxiv a license to display the preprint in perpetuity. It is made available under aCC-BY-NC-ND 4.0 International license.

\section{Figure 5}

a

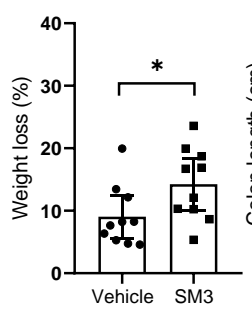

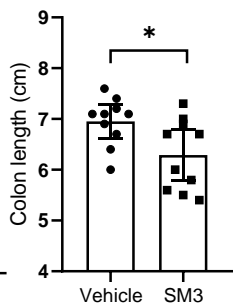

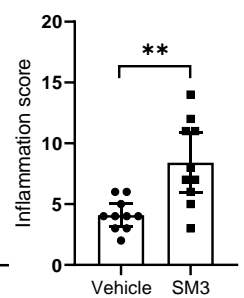

C
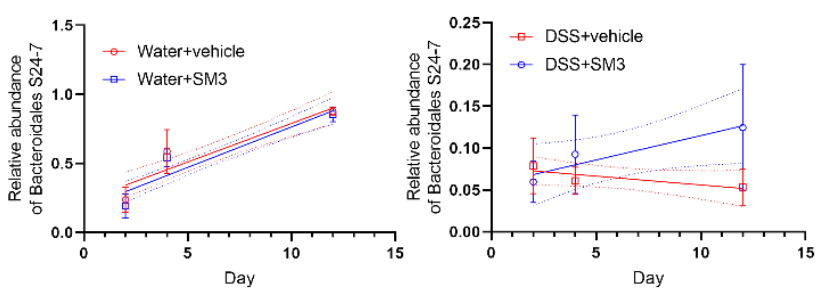

b
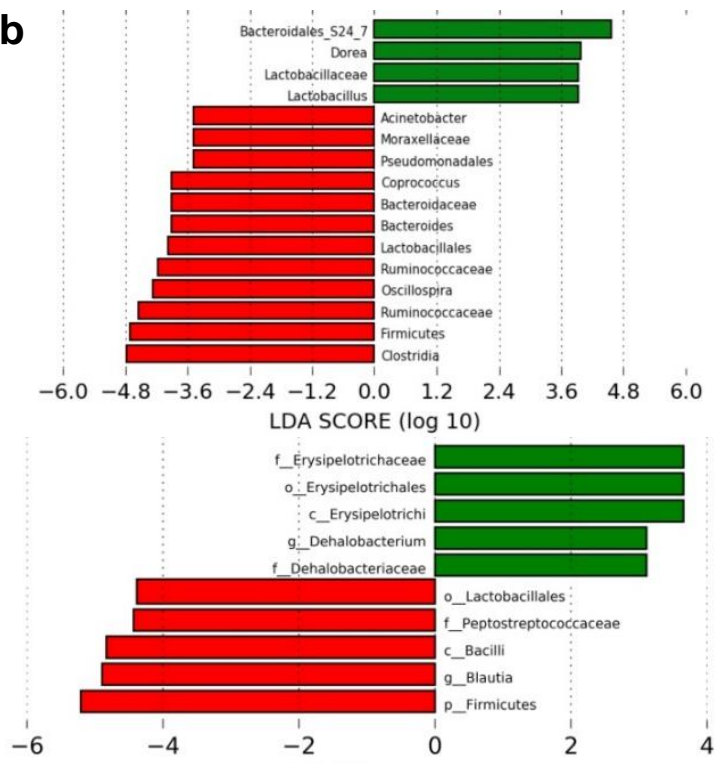

d

LDA SCORE $(\log 10)$

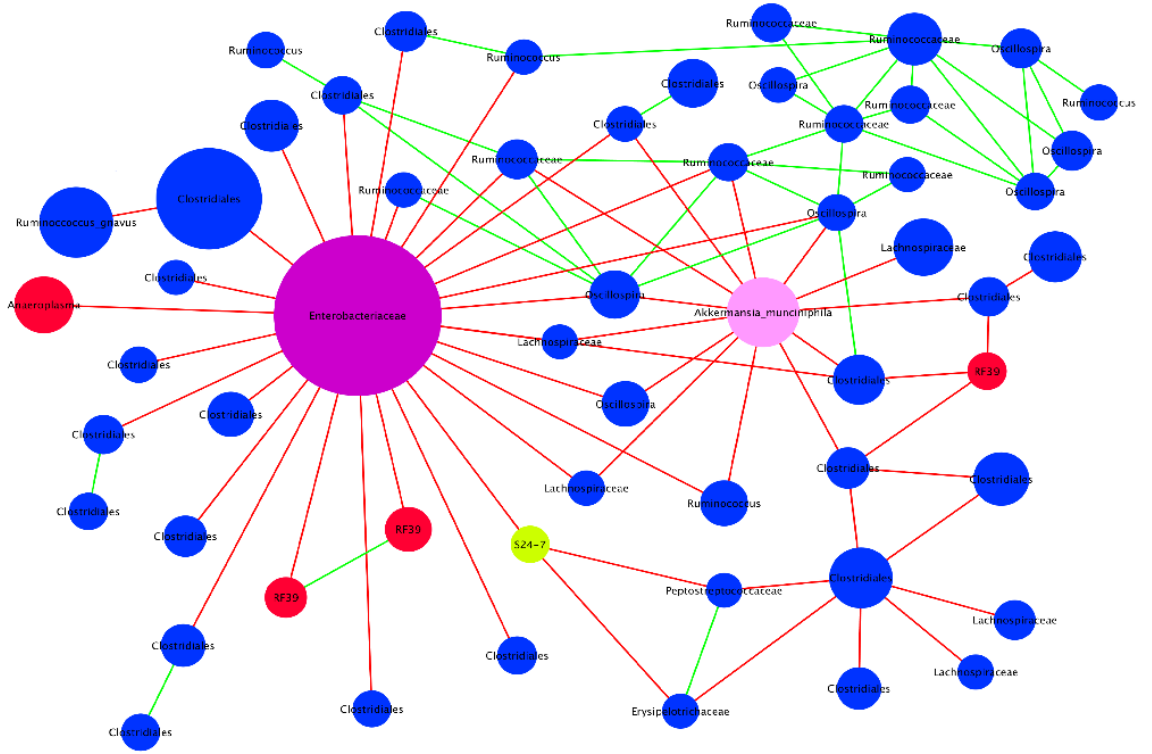

Figure $5 \mid$ Effects of SM3 on the intestinal microbiota of GF/SPF and conventional mice. a, C57BL/6 GF/SPF mice (5-week old) were exposed to DSS water and treated with vehicle (LB) or SM3 for 6 days. a indicates weight loss (left), colon length (middle), and inflammation score (Borton et al.) ( $\mathrm{n}=10$ per treatment group). b, Linear discriminant analysis (Walter et al.) Effect Size (LEfSe) plot of taxonomic biomarkers identified using feces of SM3 treated conventional $(n=10)$ (upper) and GF/SPF $(n=10)$ (lower) colitic mice on day 12 and day 6 , respectively, as compared to vehicle $(\mathrm{n}=10)$. Green and red bars indicate bacterial enrichment within SM3 treated and vehicle group respectively. All taxa that yielded an LDA score >3.0 are presented. c, Relative abundance of S24-7 in the feces from DSS (Allison et al., 1994; Borton et al.) and control (left) mice treated with SM3 or vehicle $(n=8$ per treatment group). Linear regression line was fit to show the trend of the change (dotted lines show the 95\% confidence bands). The slope of the SM3 treated group is similar to vehicle in water control group $(P=0.7827)$, but significantly different in DSS group $(P=0.0182)$. d, Co-occurrence network plot showing strong positive and negative correlations between OTU abundances. All networks were generated with CoNet and visualized in Cytoscape. Processing was applied to the dataset with CoNet. Input filtering constrained the minimum occurrence of OTUs and considered only those present in at least $50 \%$ of samples. Standardization normalized dataset columns. Networks were constructed using Spearman's correlation methods with threshold setting at 0.9 , Bray Curtis dissimilarity at the automatic threshold setting, and Kullback-Leibler dissimilarity at the automatic threshold setting; the edge selection parameter was set to 30 for the strongest positive and negative correlations. Randomization steps included permutations and bootstraps with filtering of unstable edges and Benjamini-Hochberg procedure with a P-value of 0.05 . Node clusters with less than or equal to three edges were not shown in the final network. Edge coloration indicates copresence in green or mutual exclusion in red. Nodes were colored by taxonomic phylum and labeled by the highest taxonomic ranking available.Unless otherwise noted, data are represented as mean and $95 \%$ CI, and significance tested using a two-tailed Student's t-test. OTU, Operational Taxonomic Unit; GF/SPF, Germ-Free mice transferred to specific pathogen free conditions. 
bioRxiv preprint doi: https://doi.org/10.1101/759886; this version posted October 7, 2020. The copyright holder for this preprint (which was not certified by peer review) is the author/funder, who has granted bioRxiv a license to display the preprint in perpetuity. It is made available under aCC-BY-NC-ND 4.0 International license.

a

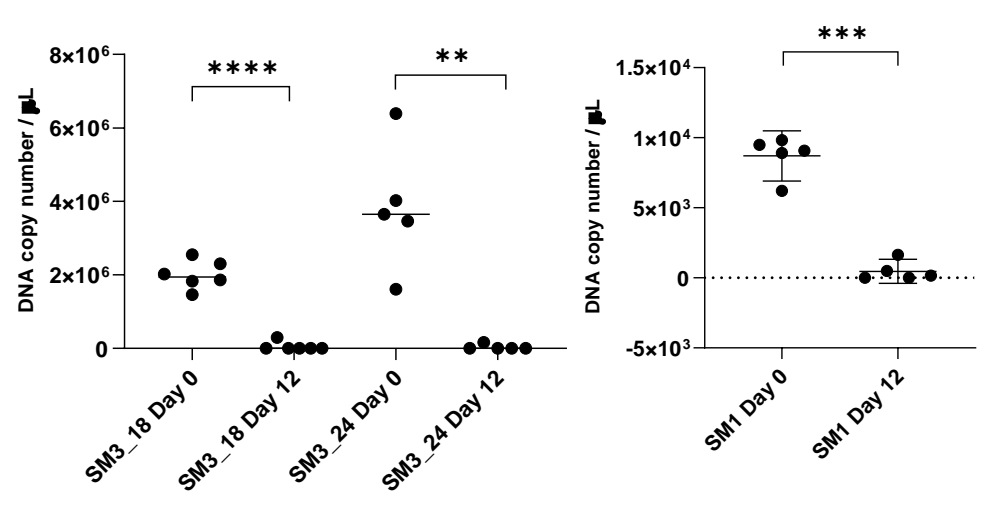

b

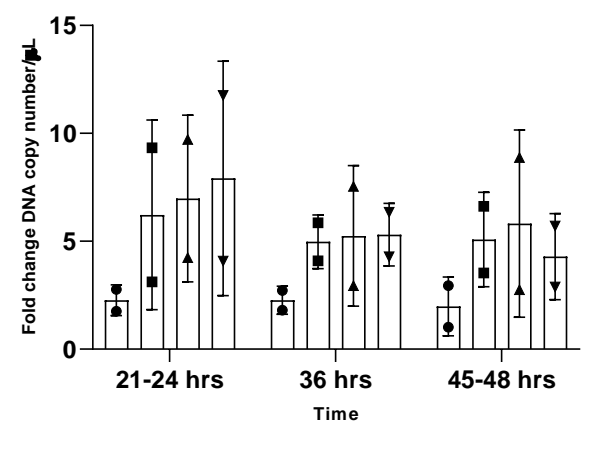

- M. intestinale only

- $\mathrm{SM} 3$

- SM3_18

SM1

C

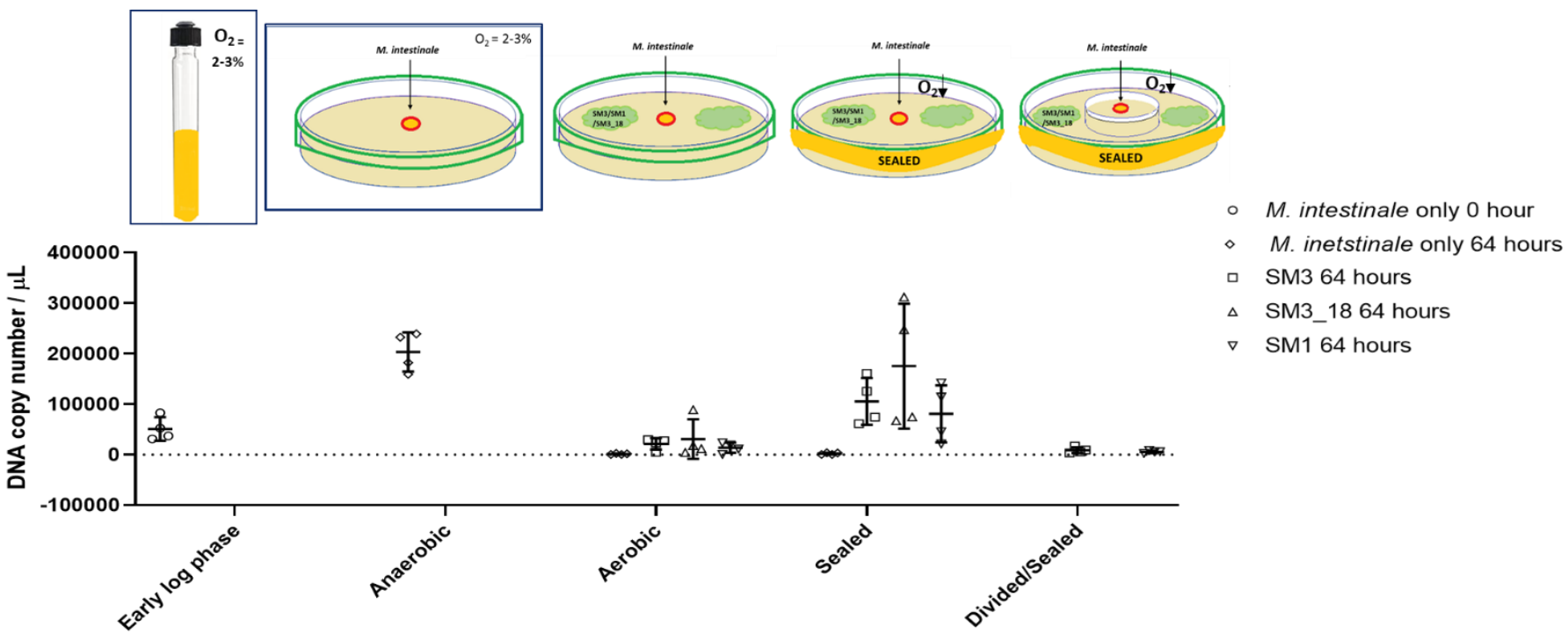

Figure 6 | Effect on S24-7 levels in the presence of SM3 and the insufficient (or inefficient) swarming variants in vivo and in vitro. a, Eight (8)-week old mice ( $\mathrm{n} \geq 5$ per treatment group) were exposed to DSS water and treated with SM3_18, SM3_24, and SM1 by oral gavage for 12 days. Total DNA was extracted from feces collected on day 0 and day 12, processed and assessed using qPCR. Five (5) ng of total DNA in conjunction with S24-7 specific primers were used to quantify bacterial copy numbers. In each assay, DNA copy number/ $\mu \mathrm{L}$ was calculated based on an internal standard curve. b-c, In vitro co-culture assay using $M$. intestinale cells grown in Chopped meat medium under anaerobic condition until early $\log$ phase $\left(\mathrm{OD}_{600} \approx 0.5\right)$ were used. $\mathbf{b}$, Fold change DNA copy number/ $\mu \mathrm{L}$ relative to $M$. intestinale monoculture. In brothbased assay, $2 \mu \mathrm{L}$ of early $\log$ phase cells of SM3, SM3_18, or SM1 was added to $M$. intestinale cells and mixed cells or monoculture of $M$. intestinale was collected at regular intervals $(21-24,36,45-48 \mathrm{hrs})$. c, In swarming-plate based assay, early $\log$ phase M. intestinale was transferred in the bore-well and SM3, SM3_18, or SM1 was allowed to swarm either under aerobic or sealed condition at $37^{\circ} \mathrm{C}$ and $\mathrm{RH} \approx 50 \%$. Plates were sealed using parafilm to create and maintain anaerobiosis due to the act of swarming. M. intestinale grown under anaerobic condition was used as a positive control. In Divided/Sealed condition, swarming region was physically separated from the bore -well containing $M$. intestinale and sealed using parafilm. Closed boxes represent incubation in an anaerobic chamber. DNA extracted from equal volume of culture and resuspended in equal volume of TE buffer was used for qPCR in conjunction with $M$. intestinale specific primers. a, Data represented as mean and $95 \% \mathrm{CI}$, and significance tested using paired t-test. b-c, Data represented as mean $( \pm \mathrm{SD})(\mathrm{n}=2$ independent experiments and 2 technical replicates for each). 


\section{Graphical Abstract}

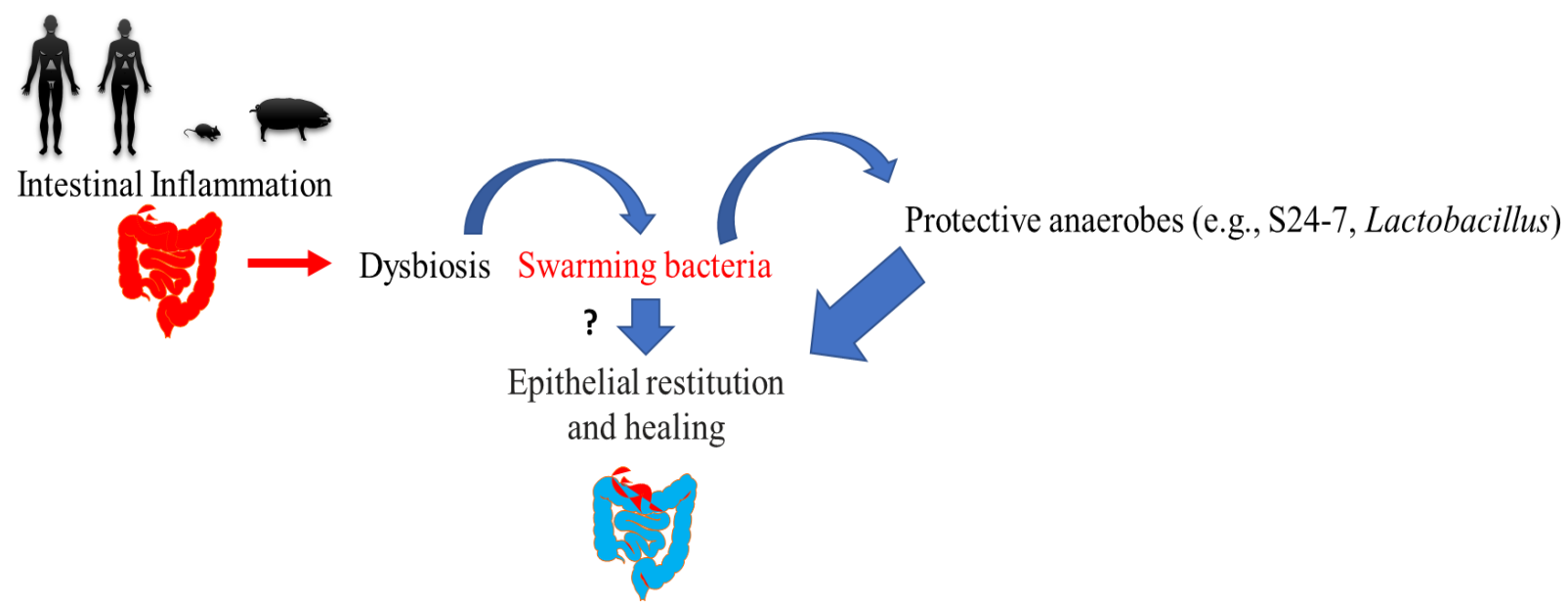

Schematic of proposed mechanism of cause and consequence of bacterial swarming during intestinal stress. Acute intestinal stress (e.g., colitis), as opposed to a homeostatic colonic lumen, induces growth of bacteria with swarming properties. If the abundance of swarming bacteria has reached sufficient levels passing some threshold CFU, it may exhibit epithelial restitution and healing by a direct or indirect mechanism and reduce local luminal oxygen levels in the intestine creating a favorable environment for enrichment of anaerobes. The bloom of protective anaerobes such as belonging to family Bacteriodales S24-7 and Lactobacillaceae in turn correlates with the accelerated resolution of inflammation and healing in colitic mice. Thus, bacterial swarming is a protective response to intestinal stress and one that is garnered during the evolution of colitis. Dashed line represents a possible unknown link, "?" represent an underlying possible direct mechanism; CFU, Colony Forming Units. 


\section{STAR Method}

479 Clinical Study. From August 2014 through January 2018, sixty-three (63) patients were 480 consented to participate in a colonoscopy aspirate or fecal collection study that was approved by 481 the Institutional Review Board (IRB) (\#2015-4465; \#2009-446; \#2007-554). The patients eligible 482 for colonoscopy were enrolled sequentially after they provided study consent (\#2015-4465; NCT 483 04089501). This study was audited by the IRB on April 24, 2019. All patients were screened and 484 consented by a single gastroenterologist and Inflammatory Bowel Disease specialist (DL). Patients 485 were enrolled if they had a diagnosis of inflammatory bowel disease (Crohn's disease or Ulcerative colitis) or were undergoing routine screening colonoscopy for colorectal polyps/cancer or required a colonoscopy as part of their medical management of any gastrointestinal disorder as clinically indicated. The following information was collected in the clinic and codified (numerically) by the gastroenterologist (DL). Laboratory personnel receiving the aspirate sample were blinded to the patient, diagnosis and therapy outcome. The results of the swarming assay were then associated by un-blinding the clinical data $(\mathrm{MB}, \mathrm{DL})$. The clinical data collected included age, gender, pathology, other clinical diagnoses, and medications taken at the time of the colonoscopy. There were no dietary restrictions or special instructions for patients to follow prior to colonoscopy except for routine fasting prior to the procedure. The colonoscopy preparative regimen (split dose polyethylene glycol) was used for all patients. Patients $(n=62$ of 63$)$ successfully underwent complete colonoscopy and aspirates were taken from the region or mucosa of pathology or from descending colon while exiting from a grossly "normal" colonoscopy. One patient had a truncated colonoscopy due to incomplete cleansing and no specimen was obtained in this case. Specimens were collected in sterile fecal specimen cups without any preservative (Fisher, $650 \mathrm{~mL}$ ), kept at $4^{\circ} \mathrm{C}$ for at most 15 minutes prior to transport to the laboratory. The specimens were then transported at ambient temperature ( 15 minutes) to the laboratory for processing. Ten (10) deidentified random frozen fecal samples from pre-screened healthy volunteers were also obtained from OpenBiome (Boston, MA) (www.openBiome.org). Glycerol can facilitate swarming of bacterial cells on soft agar medium (Kim and Surette, 2005). Samples from OpenBiome are stored in glycerol. In order to avoid any external determinant that can influence swarming of bacterial species in these fecal samples, we washed the fecal samples in sterile PBS and then incubated in

$5072 \mathrm{~mL} \mathrm{LB}$ broth overnight at $37^{\circ} \mathrm{C}, 200 \mathrm{rpm}$. Swarming assay was performed using these revived fecal cultures. Qualitative scoring of the swarmers in the clinical specimens was made based on 
the detection of bacterial spread with surfactant rim over a 72 hours incubation period. Samples showing swarming were scored as $\leq 24$ hours (Score 3), 24-48 hours (Score 2) and 48-72 hours

511 (Score 1). Samples that didn't show swarming over 72 hours of incubation were considered as “non-swarmers" (Score 0).

513 Isolation and identification of bacterial swarmers from feces. Swarming assay was performed 514 on Luria Bertani (LB) swarming agar medium (10 g/L tryptone (Sigma), $5 \mathrm{~g} / \mathrm{L}$ yeast extract 515 (Acumedia) $10 \mathrm{~g} / \mathrm{L} \mathrm{NaCl}$ (Fisher), $5 \mathrm{~g} / \mathrm{L}$ Agar (RPI)) with some modifications to an established 516 method (Morales-Soto et al., 2015). To isolate a singular dominant swarmer from a polymicrobial 517 mix of bacteria (such as feces), we initially focused on developing an assay to isolate swarmers 518 using known polymicrobial mixed cultures of bacteria. Single bacterial species (up to seven strains 519 belonging to different taxa) grown in $\mathrm{LB}\left[\mathrm{OD}_{600}\right.$ of 1.0-1.3] were mixed in a 1:1 ratio and, $5 \mu \mathrm{L}$ of 520 this mix was spotted on $0.5 \%$ agar plates. Following air drying at room temperature, the plates 521 were and incubated at $37^{\circ} \mathrm{C}, 40 \% \mathrm{RH}$ (relative humidity) for 10 hours. Bacterial swarm front was 522 swabbed using a sterile tooth-pick from the edge of swarming colony at different locations (see 523 arrows, Fig. S2) and after re-streaking on separate agar plates and scaled by growth in LB, samples 524 were identified using Matrix Assisted Laser Desorption and Ionization-Time of Flight (MALDI525 TOF). Swarmers or hyperswarmers present in the fecal or colonoscopic samples were isolated and 526 determined using an identical approach. Fecal pellets and/or colonoscopy aspirates from the clinic and/or feces of mice and pigs were collected in sterile tubes, and freshly prepared and used for

528 swarming assays before freezing in small aliquots at $-80^{\circ} \mathrm{C}$. Feces from stressed intestinal model 529 of pigs (Munyaka et al., 2016) were obtained on dry ice from Ehsan Khafipour at University of 530 Manitoba Winnipeg, Canada. Five (5) $\mu \mathrm{L}$ of homogenized feces in Phosphate buffered saline PBS, $531 \mathrm{pH} 7.2(100 \mathrm{mg} / \mathrm{mL})$ or colonoscopic aspirates were spotted on swarming agar plates (optimized 532 to $0.5 \%$ agar) and were incubated at $37^{\circ} \mathrm{C}$ and $40 \% \mathrm{RH}$, for 120 hours. Most bacterial swarmers, 533 however, were detected within the first 48-72h from incubation. Dominant swarmers from the edge 534 of the colony were identified using MALDI-TOF. Once identified, cells from the same aliquot 535 were plated on to $1.5 \%$ LB agar and serially passaged from a single colony to obtain a pure culture 536 of the strain.

537 Bacterial swarming and time-lapse imaging. Swarming ability of a single bacterial species using 538 a pure culture of Enterobacter sp. SM1 and its isogenic mutant, Enterobacter sp. SM3 and its 
transposon mutants, Serratia marcescens Db10 and JESM267, clinical isolate of Serratia marcescens, Bacillus subtilis 3610 and its isogenic mutant DS215 was always determined on LB swarming agar at $37^{\circ} \mathrm{C}$ and $40 \% \mathrm{RH}$ prior to any experiments using these strains. B. subtilis 3610 and its isogenic mutant were compared on LB swarming agar containing 0.7\% agar (Kearns and

543 Losick, 2005). Briefly, $2 \mu \mathrm{L}$ of an overnight culture grown in LB medium from a glycerol stock

544 was spotted on swarming agar plate, followed by air drying at room temperature and incubating 545 the plate overnight as stated above. Freshly made swarming agar plates, no more than 12 hours old 546 when stored at $4^{\circ} \mathrm{C}$, were used throughout the study. In order to capture real time swarming motility, a temperature and humidity-controlled incubator equipped with time lapse photography was built (see accompanying publication on Nature Protocol Exchange for detailed protocol, doi:10.21203/rs.2.9946/v1). As swarming is dependent upon RH (Kearns, 2010), we used an optimized RH of $40 \%$ that allowed image capturing without condensation on the lid of the Petridish. Unless otherwise stated, swarming potential of isogenic strains was always compared on the same swarming agar plate to nullify the difference due to the condition of the medium, which may vary between plates. Swarming area was calculated using a python-based script (available online, via Nature Protocol, doi:10.21203/rs.2.9946/v1) to identify the swarming edge using the time-lapse images. Swarming under anerobic condition $(0.1 \mathrm{ppm})$ and $10 \%$ oxygen (3.3 ppm) were performed at $37^{\circ} \mathrm{C}$ and $40 \% \mathrm{RH}$ in an anaerobic chamber (Coy Labs Inc.). In order to compare swarming motility between two strains, bacterial cultures were inoculated on a single plate and the colony areas were measured when the faster swarmer has covered half of the agar plate.

Dextran Sulfate Sodium (DSS) induced acute colitis in conventional and gnotobiotic mice. were purchased and co-housed for acclimatization at The Albert Einstein College of Medicine vivarium for 2 weeks prior to randomization by coin toss as previously described (Venkatesh et al., 2014). To induce acute colitis, mice were administered 3\% (w/v) DSS (MW 36-50 KDa) (MP Biomedicals, LLC; Cat. no. 160110) in animal facility drinking water throughout the course of the experiment. By contrast, the control group or the normal mice always received animal facility drinking water. To determine the effect of swarming and swarming deficient strains during colitis, mice were orally gavaged with $100 \mu \mathrm{L}\left(\sim 4 \times 10^{9} \mathrm{CFU} / \mathrm{mL}\right)$ test bacteria or $\mathrm{LB}$ as vehicle, daily for 
570 absolutely required use of unwashed bacterial strains grown in fresh LB (OD $600 \sim 1.0)$. In doseoptimization studies, bacterial dilutions were prepared in its own spent medium to vary cell number as the only determinant factor. Mice underwent daily monitoring for body weight, clinical signs and symptoms (e.g., occult blood, diarrhea, activity), gross water consumption (measuring water marked level), and visual inspection of rectal mucosa. At the end of the experiment, mice were euthanized using isoflurane anesthesia and intestines harvested for histopathology. The histology slides were prepared using a swiss role technique of intestines embedded in paraffin as previously published (Whittem et al., 2010). Scoring of inflammatory pathology was based on a published reference with minor modifications (Erben et al., 2014). The experimenter was not blinded to treatment allocation; however, the pathologist (K. S.) evaluating histologic scores was blinded to treatment allocation. All studies were approved by the Institute of Animal Studies at the Albert Einstein College of Medicine, INC (IACUC \# 20160706 and preceding protocols).

Colitis was induced in five-week old germ-free (GF) wildtype (WT) C57BL/6 mice under specific pathogen free (SPF) conditions (McCafferty et al., 2013) using 3\% DSS in drinking water and treated with $100 \mu \mathrm{L}\left(\sim 4 \times 10^{9} \mathrm{CFU} / \mathrm{mL}\right)$ of test strain or $\mathrm{LB}$ as vehicle for 7 days when most mice had $>10 \%$ weight drop. Mice were euthanized by $\mathrm{CO}_{2}$ asphyxiation and histology specimens were prepared using swiss roll technique. Scoring of inflammatory pathology was based on a published reference with minor modifications (Erben et al., 2014). Inflammation score and Trichrome Fibrosis score was calculated based on published references (Ding et al., 2012). All GF mouse protocols were approved by the Institutional Animal Care and Use Committee of the University of Florida (IACUC\#201308038). The experimenter (ZH, CJ) was not blinded to treatment

591 allocation; however, the pathologist (KS) evaluating histologic scores was blinded to treatment 592 allocation. In addition, a second pathologist (QL), randomly re-read a subset of the colitis histology 593 slides which had a high correlation with original pathologist read (KS) (Spearman $\rho=0.96,95 \%$ CI $0.92-0.98, P<0.0001$, two-tailed). in colitis, C57BL/6 mice were administered 3\% DSS in drinking water for 7 days (when most mice had a weight loss $>10 \%$ of their pre-DSS exposure weight). Subsequently, mice received animal facility drinking water without DSS and were further randomized by coin-toss to a treatment group delivered $4 \times 10^{9} \mathrm{CFU} / \mathrm{mL}$ of bacterial cells or LB by oral gavage for 5 days. Colon samples were 
600 prepared for Hematoxylin-Eosin (H\&E) staining and histology and processed as described above.

601 The precise number of mice used for each experiment are stated in the Figure legends and are

602 visible as separate plots in the graph. In the preliminary experiments (see Fig. $2 \mathrm{~d}$ and $7 \mathrm{c}$ ), the

603 computed means ( \pm SD's) of the inflammation score for vehicle $[7.28-9.28(+1.77-2.4)]$ and

604 wildtype bacteria gavaged mice [2.59-5.66 (+ 1.31 - 3.2)] allowed for determination of the D

605 value ( ranging between $2-3$ ). The exact sample size was determined under consideration of the

606 D value, available mice per order, and ethical aspects (implementing replication studies, use of

607 coin toss 1:1 randomization, and cage space at any given time point) as well as an assumed

608 estimated inflation factor of $\leq 10 \%$. For certain experiments, if insufficient number of mice were

609 available for a reliable significance prediction, biologically independent repetition experiments

610 were performed and data pooled for analysis (e.g., Fig. 2a-f, Fig. 7a-e).

611 Construction of transposon mutants. In order to generate an isogenic swarming deficient strain

612 of SM3, we adopted an in vivo transposition approach using pSAM_Ec with some modifications

613 (Wiles et al., 2013).pSAM_Ec was a gift from Matthew Mulvey (Addgene plasmid \#102939;

614 http://n2t.net/addgene:102939; RRID: Addgene_102939). In short, donor strain -

615 EcS17/pSAM_Ec was grown from an overnight culture in pre-mating medium (M9 salts

616 containing $40 \mu \mathrm{g} / \mathrm{mL}$ threonine and proline, $1 \mu \mathrm{g} / \mathrm{mL}$ of thiamine) with $0.2 \%$ glucose until mid-

617 exponential phase $\left(\mathrm{OD}_{600}\right.$ 0.5-0.6). Similarly, the recipient strain SM3 was grown in pre-mating

618 medium containing $0.4 \%$ lactose until early exponential phase $\left(\mathrm{OD}_{600}\right.$ 0.2-0.3). After heat shock

619 treatment of the recipient strain at $50^{\circ} \mathrm{C}$ for 30 minutes the cell density of SM3 was scaled up to

620 obtain similar number of cells as that of the donor strain. For conjugation, $750 \mu \mathrm{L}$ of both the

621 strains were mixed, the cells were washed twice in M9 salts and re-suspended again in the same

622 medium. The mixture of donor and recipient was placed on a sterile $0.45 \mu \mathrm{m}$ membrane disc

623 (Millipore) rested on mating agar plate (1x M9-thr-pro-thi-glucose agar) and incubated upright at

$62437^{\circ} \mathrm{C}$ overnight. Next day, the cells were dislodged from the membrane in M9 medium by

625 vortexing and plated on selective agar medium (1x M9-threonine-glucose-kanamycin agar)

626 containing kanamycin. Individual colonies were spotted on LB swarm agar plate to screen non-

627 swarming or swarming deficient isogenic strain of SM3. The presence of transposon was

628 confirmed by using transposon specific primer while the location of transposon insertion was

629 verified by APPCR (Saavedra et al., 2017) followed by Sanger sequencing and mapping into the

630 SM3 genome (STAR Method Table 1). 
16S rRNA profiling to identify shift in colon microbiome. $16 \mathrm{~S}$ rRNA meta-analyses of the fecal samples from mice were conducted at Wright Labs, LLC. Fecal samples were shipped to Wright Labs, LLC on dry ice, and underwent DNA isolation using a Qiagen DNeasy Powersoil DNA Isolation kit following the manufacturer's instructions (Qiagen, Frederick, MD). DNA was quantified and checked for its quality using the double stranded DNA high sensitivity assay on the Qubit 2.0 Fluorometer (Life Technologies, Carlsbad, CA). The 16S rRNA gene was amplified using Illumina iTag Polymerase Chain Reactions (PCR) based on the Earth Microbiome Project's 16S rRNA amplification protocol (Walters et al., 2015). Amplified DNA was pooled, gel purified at $400 \mathrm{bp}$ and multiplexed with other pure libraries to form a sequencing library normalized to the final concentration of library observed within each sample. The sequencing library was sequenced using an Illumina MiSeq V2 500 cycle kit cassette with 16S rRNA library sequencing primers set for 250 basepair (bp) paired-end reads at Laragen Inc (Culver City, CA).The paired-end sequences were merged with a minimum overlap of 200 bases, trimmed at a length of $251 \mathrm{bp}$, and quality filtered at an expected error of less than 0.5\% using USEARCH (Edgar, 2010). The reads were analyzed using the QIIME 1.9.1 software package (Caporaso et al., 2010; Caporaso et al., 2011). Chimeric sequences were identified and assigned operational taxonomic units (OTU) using UPARSE at 97\% identity (Edgar, 2013). The taxonomy was assigned using the Greengenes 16S rRNA gene database (13.5 release) (DeSantis et al., 2006). Linear discriminant analysis Effect Size (LEfSe) analysis was conducted to identify significantly enriched taxa within categorical groups of interest (Segata et al., 2011). For all comparisons, a Kruskal-Wallis alpha $(\alpha)$ was set at 0.05 to identify significantly enriched taxa, and a pairwise Wilcoxon rank sum test was utilized to test biological consistency across all subgroups $(\alpha=0.05)$. Linear discriminant analysis (Walters et al., 2015) was calculated to determine effect size, and the 5 most strongly enriched taxa within each cohort were plotted. Co-occurrence network analysis was conducted on an unrarified OTU table containing bacterial abundance data from DSS+ SM3 treated samples and created within the Cytoscape plugin Conet (Faust and Raes, 2016); (Shannon et al., 2003). A spearman's rho threshold of $\mid 0.7$ | was implemented prior to network plotting.

659 Bacterial cells picked from the leading edge of the swarming colony within the surfactant layer 660 were sub-cultured on LB and then repeatedly sub-cultured onto MacConkey II, Columbia 661 Nalidixic Acid (CNA) with 5\% sheep blood, and Trypticase Soy Agar with 5\% sheep blood plates 
662 (Becton Dickinson, Sparks, MD). Culture plates were incubated overnight at $35^{\circ} \mathrm{C}$. A minimum 663 of three colonies from each sample were identified by MALDI-TOF analysis using a MALDI 664 Biotyper (Bruker Daltonics, Billerica, MA) in conjunction with Real Time Classification software 665 (Bruker Daltonics, version 3.1). When evident, colonies with varied lactose fermentation reactions 666 and/or colony morphologies were chosen for MALDI identification. Colonies were identified by 667 directly transferring the bacteria to a MALDI target plate followed by the addition of $70 \%$ formic 668 acid (Sigma-Aldrich, St. Louis, MO) and HCCA ( $\alpha$-cyano-4-hydroxycinnamic acid) matrix 669 (Bruker Daltonics). When necessary, colonies with low MALDI identification scores (0-1.999) 670 were sub-cultured, and a tube-based extraction was performed to attempt to improve the 671 identification score. Briefly, colonies were added to $300 \mu \mathrm{L}$ of water (Sigma-Aldrich) and 672 emulsified followed by the addition of $100 \%$ ethanol (Sigma-Aldrich). The bacterial suspension 673 was centrifuged (13,000 rpm, 2 minutes, RT) and the supernatant removed from the bacterial 674 pellet. To extract the bacterial proteins, $50 \mu \mathrm{L}$ of $70 \%$ formic acid and $50 \mu \mathrm{L}$ of acetonitrile (Sigma675 Aldrich) were added to the bacterial pellet, the sample was vigorously vortexed, and again 676 centrifuged. The supernatant $(1 \mu \mathrm{L})$ was spotted onto MALDI targets in triplicate for 677 identification. MALDI identification scores of 1.7-1.999 were considered indicative of a reliable 678 genus level identification whereas a MALDI score $\geq 2.0$ indicated reliable genus and species, 679 unless otherwise indicated.

680 Bacterial strains and medium. All the bacterial strains used in this study were grown in LB broth $681(10 \mathrm{~g} / \mathrm{L}$ tryptone, $5 \mathrm{~g} / \mathrm{L}, 10 \mathrm{~g} / \mathrm{L} \mathrm{NaCl})$ at $37^{\circ} \mathrm{C}$ with agitation at $200 \mathrm{rpm}$, unless otherwise stated. 682 Gram staining of SM1 and SM3 was performed using a commercially available Gram Staining kit 683 (Becton Dickinson Microbiology Systems, Sparks, Maryland, USA) according to established 684 protocol. For swarming, swimming and growth curve assays, bacterial strains were always grown 685 from a glycerol stock stored at $-80^{\circ} \mathrm{C}$. Growth fitness of bacterial cells in $\mathrm{LB}$ at $37^{\circ} \mathrm{C}$ when grown 686 with agitation was assessed spectrophotometrically at $600 \mathrm{~nm}$ using Beckman Spectrophotometer 687 DU 640B. Growth curve was fitted using absorbance of culture as a function of time. Specifically, 688 for growth analysis of SM1 and SM3, growth in LB was monitored at $600 \mathrm{~nm}$ every 20 minutes 689 using a plate reader (PERKIN ELMER HTS7000) at $37^{\circ} \mathrm{C}$ for 24 hours.

690 Measurement of microlevels of oxygen in mouse lumen. Oxygen concentration in the mouse 691 lumen was assessed using a profiling oxygen microsensor (PresensIMP-PSt7-02) with a flat tip 
692 that has the ability to detect in the range of $0-1400 \mu \mathrm{M}$ oxygen with an accuracy of $\pm 3 \%$. The

693 control or DSS treated mice were first anesthetized in isoflurane for at least 3 minutes, and then

694 the microsensor probe was inserted from the anal verge. The oxygen concentration was monitored

695 for one minute at different locations across the colon $(0.5,1$ and $2 \mathrm{~cm}$ from the anus) using "Presens

696 Measurement Studio 2 (version 3.0.1.1413)”. In order to avoid damage of the probe and mucosa

697 while inserting through the anus, we used an Ethylenetetrafluoroethylene (ETFE) tube (outer

698 diameter: $1 \mathrm{~mm}$; inner diameter: $0.7 \mathrm{~mm}$ ) to house the probe. The housing was retracted to expose

699 the probe in the designated location and cleaned before moving to the next location within the

700 colon.

701 Consumption of residual oxygen on swarming plates. Swarming plates were prepared as 702 described previously and a fine hole $(3 \mathrm{~mm} \times 1 \mathrm{~mm})$ was made on the lid of the plate to fix a 703 syringe-based oxygen microsensor probe (Presens, NTH-PSt7-02). After inoculation of the test 704 bacterial culture, the probe was inserted into the swarming agar medium through the hole on the 705 lid, finely adjusted using a manual micromanipulator (Presens) and then sealed using silicon oil. 706 The side of the Petri dish was sealed using parafilm, and this whole unit was placed in the 707 indigenously built environmental controlled incubator at $37^{\circ} \mathrm{C}$. The oxygen consumption within 708 the agar plate over time was monitored every 5 minutes for 20 hours using "Presens Measurement 709 Studio 2". The average oxygen consumption rate in a sealed container was calculated by dividing 710 the change in oxygen concentration with time at which the oxygen levels reached a plateau phase. 711 Consistently, we have observed that during swarming activity of SM3, the plateau phase stabilizes 712 at an oxygen concentration of $0.003 \mathrm{ppm}$. This validated that the system used in this study was 713 properly sealed from the outside environment.

714 Swarming on mucosal surface. We used colon tissue from mice that had received 3\% DSS water 715 or water for 10 days to develop a mucosal race experiment. Normal or DSS treated mice were 716 euthanized, and the large intestines were cut open and cleaned to remove residual feces. After 717 rinsing thoroughly twice in $35 \%(\mathrm{v} / \mathrm{v})$ ethanol and PBS, the intestines were sectioned into small 718 segments of around 1.5-2.5 cm each. A hybrid plate with sterile swimming agar (3 g/L) and hard 719 agar $(15 \mathrm{~g} / \mathrm{L})$ was prepared, where one half of the plate had $1.5 \%$ agar and the other half was filled 720 with $0.3 \%$ agar containing LB. To make such hybrid agar plate, $1.5 \%$ agar was poured first and 721 once solidified half of the gel was removed using a sterilized spatula to fill the rest of the Petri dish 
722 with swimming agar. The tissue pieces were placed on 1.5\% agar in a way so as to have one end 723 of the tissue precisely overlapping with the border between 1.5\% agar and the swimming agar.

724 Overnight bacterial cultures were serially diluted 1012 times to reach cell concentration of 106 $725 \mathrm{CFU} / \mathrm{mL}, 2 \mu \mathrm{L}$ of which was inoculated on a $2 \mathrm{~mm} \times 2 \mathrm{~mm}$ sterilized filter membrane (MF726 Millipore, $0.45 \mu \mathrm{m}$ ). Bacterial cells adsorbed on membrane was then used as a source of inoculum 727 on the mucosal surface. This avoided wetting of tissue surface that may facilitate free swimming 728 and free flowing of bacterial cells on tissue surface. The motility of a swarming deficient and its 729 wild type was always compared using a piece of tissue that belonged to the same region of the 730 colon in mice. The plates were dried in the laminar hood for 20-30 minutes before incubating at $73137^{\circ} \mathrm{C}$ and $40 \% \mathrm{RH}$ overnight. Drying of plates allowed removal of excess moisture from the 732 topmost layer of the tissue. Time-lapse photos were captured to evaluate the time at which bacterial 733 test strain reached the other end of the intestinal tissue indicated by the swimming of bacteria on $7340.3 \%$ LB agar. Distance travelled by the bacterial strain was measured in ImageJ according to the 735 pixel/length ratio. The motility rates were calculated as Distance travelled / Time duration in which 736 the test strain reached the swim agar.

737 Swimming assays. Free swimming of bacterial cells was observed in fresh cultures that were 738 grown in LB from an overnight culture (1:100 dilution in fresh LB) until $\mathrm{OD}_{600} \sim 0.3$. At this point, 739 cells were further diluted in PBS (1:50) and spotted on a glass slide with a cover slip placed on top 740 of it. Swimming cells were observed under a phase contrast microscope (OMAX M837ZL, 40X) 741 and videos captured using software (OMAX ToupView 3.7). The videos were captured at a frame 742 rate of $18 \mathrm{fps}$ for $\sim 1$ second for each trajectory and then processed in Image (ver. 1.52g) to analyze 743 swimming speeds of the test bacterium. Ten (10) straight trajectories of motion were picked 744 randomly and the average speed was calculated as Trajectory length/Time.

745 For soft-agar swimming assay that may be relevant to in vivo conditions, $2 \mu \mathrm{L}$ of overnight culture 746 of the test bacterium was inoculated on LB swimming plate $(10 \mathrm{~g} / \mathrm{L}$ tryptone, $5 \mathrm{~g} / \mathrm{L}, 10 \mathrm{~g} / \mathrm{L} \mathrm{NaCl}$, $7473 \mathrm{~g} / \mathrm{L}$ Agar) and incubated at $37^{\circ} \mathrm{C}, 40 \% \mathrm{RH}$ in our indigenously made incubator. To compare 748 swimming potential of isogenic mutants, the isogenic pairs were inoculated on the same swim agar 749 plate. Captured image in which the swimming colonies have not merged in due time, were used to 750 calculate swim area using "Free selection" tool in ImageJ (ver. $1.59 \mathrm{~g}$ ). 
751 Genome sequencing, assembly, and annotation. We used a combination of short and long read 752 sequencing to sequence and assemble two strains, SM1 and SM3. Short read library construction 753 and sequencing was done at the New York Genome Center using a 2×300 paired end MiSeq run. 754 Because we had many contigs for each strain, we next did PacBio® Single Molecule Real Time 755 (SMRT) long read sequencing to improve our assemblies. SMRT sequencing was done at the Yale 756 Center for Genome Analysis. The Hierarchical Genome Assembly Process (HGAP) was used for 757 assembly of the genomes. SMRT sequencing and HGAP assembly yielded one contig for each 758 strain. We then did a combined assembly with the PacBio and Illumina data using SPAdes (version 759 3.6.2) (Bankevich et al., 2012), using the PacBio contig with the 'trusted_contigs' parameter for 760 each strain with otherwise default parameters. The CLC command clc_mapper (version 4.4.2.133896) was used to assess the quality of the final assembly for each strain. The final assembly for each strain was annotated using Prokka (version 1.3) (Seemann, 2014) annotating as "Bacteria" with otherwise default parameters.

Genome comparisons. The SM1 and SM3 strains were compared to other available bacterial genomes using three approaches: 1) comparing the SM1/SM3 16S rRNA gene sequences to a large database of bacterial 16S sequences; 2) comparing conserved genes in SM1/SM3 to conserved genes in other bacterial genomes using multi-locus sequence typing (MLST); and 3) whole genome BLAST comparisons. Prokka identified eight 16S rRNA gene sequences in each strain. For 16S based analysis, these sequences were input to the Silva ACT: Alignment, Classification and Tree Service (Yilmaz et al., 2014). We used the "search and classify" and "compute tree" options with default parameters excepting that we restricted our search to one neighbor per query sequence. For MLST analysis, we input the SM1 and SM3 strains to autoMLST (Alanjary et al., 2019), an automated webserver that uses a precomputed set of marker genes to generate a multi locus species

774 tree that placed SM1 and SM3 with their closest genomic neighbors. Both 16S and MLST analysis identified SM1 and SM3 as Enterobacter strains. We therefore used the BLAST Ring Generator (BRIG) (Alikhan et al., 2011) to compare the complete genomes of SM1 and SM3 with two 777 clinically important close neighbors, Enterobacter cloacae subsp. cloacae ATCC 13047 and 778 Enterobacter asburiae strain ATCC 35953. To run BRIG (Alikhan et al., 2011), we used the 779 longest contig from SM1 (5107194 bp) as the reference genome and compared the other three 780 genomes (SM3, E. cloacae, and E. asburiae) to SM1. 
781 Quantification of bacteria in feces using qPCR. To estimate species specific bacterial abundance

782 (SM3, SM3_18, SM3_24, B. subtilis 3610 and DS215, S. marcescens Db10 and JESM267) present

783 in murine feces, we approached a qPCR-based assay using total microbial DNA extracted from

784 feces. DNA was extracted from feces samples collected on Day 0, 4 and 10 using DNeasy

785 Powersoil kit (Qiagen). In case of glycerol stocks of feces, stocks were pelleted at 5,000 x g for 8

786 minutes, glycerol removed and re-suspended in $400 \mu \mathrm{L}$ PBS solution. A total of $400 \mu \mathrm{L}$ of the

$787 \mathrm{PBS} /$ bacteria solution was then added to the lysing tube provided with the kit. SM3 specific

788 primers (Just_F1 and Just_R1, Supplementary Table 2) were designed that did not show any 789 amplification with E. coli DH5 $\alpha$ and Enterobacter cloacae (the nearest neighbors by MLST).

790 Bacillus subtilis and Serratia marcescens specific primers were adapted from previous studies

791 (Bussalleu and Althouse, 2018; Fall et al., 2004). qPCR was performed using either Sensifast kit

792 (Bioline) or PowerUp SYBR Green master mix (Applied Biosystem's) following manufacturer's

793 protocol. Quantification of amplicon (copy number/ $\mu \mathrm{L}$ ) was calculated based on a standard curve

794 generated using amplicon of interest as template DNA.

795 Construction of isogenic mutants. Isogenic mutants of either Enterobacter sp. SM1 or

796 Salmonella enterica serovar typhimurium were constructed using recombineering and PCR

797 Ligation mutagenesis approach (Datta et al., 2006; Lau et al., 2002). Briefly, red recombineering

798 plasmids pSIM5 or pSIM6 (graciously donated by Donald Court at National Cancer Institute, MD,

799 USA) was electroporated in electrocompetent cells of SM1 or Salmonella (Electric field strength

$80012.5 \mathrm{KV} / \mathrm{cm} \& 200 \Omega$ ) using Gene Pulser (Biorad). Bacterial cells harboring these plasmids were 801 grown in LB from an overnight culture until the cell density reached OD 600 0.4-0.5. At this point

802 Red recombinase proteins were induced by further incubating the cells at $42^{\circ} \mathrm{C}$ for 15 minutes

803 followed by transferring the cells on ice for additional 15 minutes in order to make the cells

804 electrocompetent. To make gene specific deletion a gene cassette containing FRT-Kan-FRT

805 fragment or kanamycin resistance marker flanked by upstream and downstream gene sequences of

806 the target gene was generated. Upstream and downstream regions were PCR amplified from 807 genomic DNA, while FRT-Kan-FRT was amplified from the plasmid pKD4 as a template, using 808 gene specific primers (Supplementary Table 2). Kanamycin resistance marker without FRT 809 flanking region was amplified from pCAM48 (Hooven et al., 2016). Restriction digestion, ligation 810 and PCR amplification using primers that can anneal at the 5' end of the upstream region and 3' 811 end of the downstream region (upstream forward and downstream reverse primers) generated a 
812 gene cassette that can facilitate homologous recombination. Purified DNA was electroporated in

813 bacterial cells in which lambda recombinase was expressed, followed by revival of cells in SOC

814 medium at $30^{\circ} \mathrm{C}$ for 2 hours before selection of the clonal mutants on LB Agar containing 75

$815 \mu \mathrm{g} / \mathrm{mL}$ kanamycin during an overnight incubation. At least two colonies were selected and

816 transformed with flippase encoding pCP20 (Cherepanov and Wackernagel, 1995) in order to

817 facilitate markerless gene deletion. Homologous recombination followed by excision of

818 kanamycin resistance gene at the loci of interest was verified by Sanger sequencing.

819 Qualitative measurement of surfactant production using blood agar hemolysis. Blood agar 820 hemolysis assay was optimized based on previously established method (Walter et al., 2010). 821 Briefly, $10 \mu \mathrm{L}$ of overnight culture was inoculated on $15 \mathrm{~mL}$ Columbia Base agar plate 822 supplemented with $2.5 \%$ defibrinated sheep blood. All the plates were incubated for $48 \mathrm{~h}$ at $37^{\circ} \mathrm{C}$, 823 unless otherwise stated. The area of the zone of hemolysis was calculated using "Oval selection" 824 tool in ImageJ.

825 Qualitative measurement of surface tension using drop-collapse assay. Overnight bacteria 826 culture was spun down at 4,500 rpm for 4 minutes. $5 \mu \mathrm{L}$ of bacteria supernatant were pipetted on 827 a polystyrene 96-well plate lid (Falcon). The droplet was placed at the center of a well and the lid 828 was scanned (Bodour and Miller-Maier, 1998). Cross-sectional area of the droplet was calculated 829 using "Free selection" tool in ImageJ.

830 Qualitative measurement of surface tension using drop-counting assay. Drop-counting assay 831 was performed with some modifications to an established method (Dilmohamud et al., 2005). 832 Overnight bacteria culture was spun down at 4,500 rpm for 4 minutes. A glass Pasteur pipet was 833 then used to transfer the supernatant to a plastic cuvette drop by drop. Number of droplets was 834 counted for the spent medium supernatant to reach a total volume of $1 \mathrm{~mL}$.

835 Cytotoxicity of DSS on model intestinal human epithelia in the presence or absence of viable 836 SM3 cells. To verify if SM3 can breakdown DSS affecting its cytotoxic efficacy, we treated Caco8372 cells (ATCC HTB-37), a colorectal adenocarcinoma cell line, with DSS that was incubated with 838 SM3. Caco-2 cells were grown in complete medium: EMEM medium supplemented with 2mM L839 glutamine, 20\% Fetal bovine serum and 1X Anti-Anti (antimicrobial cocktail, Gibco) at $37^{\circ} \mathrm{C}, 5 \%$ $840 \mathrm{CO}_{2}$. When at confluence, cells were washed in sterile PBS (pH 7.2), trypsinized for 5 minutes 
841 and then resuspended in complete medium before seeding into 96 well plates. The seeding 842 concentration was 30,000 cells/well. MTS assay (Cell Titer 96 Aqueous One Solution Cell

843 Proliferation Assay, Promega) was used to determine cytotoxicity of DSS on Caco-2 cells. A dose-

844 response assay using various concentrations of DSS in cell culture medium $(8 \%-0.03 \%$, by serial

845 dilution) showed a linear relation between DSS concentration and Caco-2 cytotoxicity. We chose

846 to incubate SM3 cells in $8 \%$ DSS-medium. Bacterial cells $\left(10^{8} \mathrm{CFU} / \mathrm{mL}\right)$, obtained from a fresh

847 culture in $\mathrm{LB}\left(\mathrm{OD}_{600} \approx 0.1\right)$ after centrifugation followed by removal of spent medium, was re-

848 suspended in $8 \%$ DSS-medium and incubated at $37^{\circ} \mathrm{C}$ for an hour with slow agitation. To avoid 849 contamination of Caco-2 cells by SM3, the bacterial cells in DSS were heat killed at $95^{\circ} \mathrm{C}$ for 10

850 minutes and then spun down to collect the supernatant. $100 \mu \mathrm{L}$ of this supernatant was used to 851 treat Caco-2 cells grown in 96 well plate for durations of $1 \mathrm{~h}, 12 \mathrm{~h}$ and $24 \mathrm{~h}$. Similarly, heat killed $852 \mathrm{SM} 3$ (at $95^{\circ} \mathrm{C}$ for 10 minutes) prior to incubation in $8 \% \mathrm{DSS}, 8 \%$ DSS alone, SM3 in complete 853 medium, and complete medium alone were treated identically and used as controls in each assay. 854 The cytotoxic efficacy was evaluated using MTS assay. This assay, at each time point, was 855 performed in triplicate with three internal repeats. Cell survival (\%) at each time point and in each 856 condition is reported as a normalized value to the medium only at that specific time point.

857 Tissue RT-qPCR. Colon total RNA (4 mice each group) were isolated using Trizol Reagent 858 (Ambion) and reverse transcribed using High Capacity cDNA Reverse Transcription Kit 859 (Thermofisher). RT-qPCR was performed using PowerUp SYBR Green Master Mix 860 (Thermofisher) with primers of TNFa (FW: 5'-ATGAGAAGTTCCCAAATGGC-3', RE: 5'861 AGCTGCTCCTCCACTTGGTGG-3'), IL10 (FW: 5'-TGAGGCGCTGTCGTCATCGATTTC 862 TCCC-3', RE: 5'-ACCTGCTCCACTGCCTTGCT-3'), TNFR2 (FW: 5'-CAGGTTGTCTTGA 863 CACCCTAC-3', RE: 5'-GCACAGCACATCTGAGCCT-3'), IL6 (FW: 5'-GAGGTAAAAGA 864 TTTACATAAA-3', RE: 5'-CAAGATGAATTGGATGGTC-3'), and TBP (FW: 5'-ACCGT GAATCTTGGCTGTAAAC-3'， RE: 5'-GCAGCAAATCGCTTGGGATTA-3'). PCR was

866 repeated in quadruplicate. The mRNA expression was normalized to internal control, TBP (TATA867 Box Binding Protein). The entire experiment was repeated twice for reproducibility.

868 Histologic scoring of mouse intestinal tissue exposed to Salmonella. Mice with 3\% DSS (in 869 drinking water)-induced colitis exposed to S. enterica serovar Typhimurium (day 1-5) were 870 euthanized on day 5. Weights were measured every day and on day 5 intestinal tissue (SI, small 
871 intestines; colon) and liver was processed for histology (H\&E stain). Scoring of degree of intestinal

872 damage ( $\mathrm{n}=3$ slides per treatment group; 1 slide per mouse) first followed using criteria

873 established for colitis in a prior publication (Coburn et al., 2005) and consisted of determining

874 extent of involvement and severity of luminal tissue (necrotic epithelial cells, polymorphonuclear

875 leukocytes, and presence or absence of blood); mucosal lesions (extent of epithelial desquamation,

876 ulceration, crypt abscess, mono- and polymorphonuclear leukocyte infiltrates and presence or

877 absence of granulation tissue); and submucosal lesions (mononuclear leukocytes,

878 polymorphonuclear leukocyte infiltrates, and edema). The small intestines were scored in similar

879 fashion without determining crypt abscess formation which was absent. H-scores were computed

880 by multiplying the severity of the lesion with extent (in terms of \% of area involved in that slide

881 for that lesion) (Geesala et al., 2019).

882 Mucin Staining. Alcian Blue was used to stain mucin layer of intestine based on an established 883 method described elsewhere (Dong et al., 2012). Briefly, intestinal tissues were deparaffinized and 884 hydrated in distilled water. After mordanting in 3\% Acetic Acid (v/v) for 3 minutes the tissue was 885 stained in 1\% Alcian Blue in 3\% Acetic Acid ( $\mathrm{pH} 2.5)$ for 30 minutes. Next, the specimens were dehydrated in $95 \%$ Ethanol and cleaned in xylene before mounting with Poly Mount.

Lipocalin assay. LCN2 assay was performed using Mouse Lcn2/NGAL Duoset ELISA kit (R\&D 888 System, DY1857) according to manufacturer's protocol. Briefly, fecal samples were collected and resuspended in PBS to a final concentration of $100 \mathrm{mg} / \mathrm{mL}$. Samples were either diluted at a ratio of 1:10 (day 0 samples) or 1:1000 (day 10 samples) and added to precoated ELISA wells (100 $\mu \mathrm{L} /$ well). Plates were processed and read at an absorbance of $450 \mathrm{~nm}$, corrected from optical imperfections by subtracting the reads using measurements at $570 \mathrm{~nm}$, and the corrected reads were plotted against a standard curve to calculate lipocalin concentrations.

894 RNA Extraction and metatranscriptome analysis. Cells were scraped from swarming plate and 895 resuspended in 500 $\mu \mathrm{L}$ RNALater (Invitrogen) solution before freezing. Samples were sent to 896 Wright Labs LLC. for further processing and analyses. RNA was extracted using the RNeasy 897 PowerMicrobiome Kit (Qiagen). All RNA extracts were quantified using the Qubit RNA High 898 Sensitivity Kit (Invitrogen, Carlsbad, CA, USA) to confirm complete DNase treatment of the RNA 899 extracts (DNA concentration < $0.05 \mathrm{ng} / \mathrm{uL}$ ). Subsequently, approximately $100 \mathrm{ng}$ of extracted 
900 RNA was subject to NEBNext Ultra RNA Library Prep (New England BioLabs, Ipswich, MA, 901 USA) for double stranded cDNA synthesis and RNA-Seq library preparation. Quality of the final

902 library was assessed using a high sensitivity bioanalyzer chip (Agilent). The same library 903 quantification, pooling, and purification methods were used as described above. Purified 904 metatranscriptome libraries was sequenced using Illumina NextSeq. Raw read quality was 905 assessed using the program FastQC to obtain average Q scores across the read length of all 906 sequences and quality filtered using fastp. A sliding window filtration was utilized to cut reads at 907 a 4-base average Q score of 28 or lower and reads trimmed below $60 \%$ of original length were 908 discarded (Chen et al., 2018). Filtered RNA-Seq data were aligned against the SM3 genome using 909 the Rockhopper RNA-Seq analysis platform using default parameters (Tjaden, 2015). Normalized 910 expression counts were then subject to weighted Jaccard principal coordinates analyses within the 911 QIIME2 software analysis package (Hall and Beiko, 2018). Analysis of similarity (ANOSIM) test 912 statistics were calculated to assess the significance of differential clustering between categorical 913 cohorts of interest.

914 IL-10 receptor neutralization-induced chronic colitis. Mice deficient in Toll-like receptor 5 915 (Tlr5KO) were employed in this study given their heightened response to IL-10R neutralization916 induced chronic colitis (Singh et al., 2015). Colitis was induced in 8-week-old female Tlr5KO 917 mice by administering rat anti-IL-10R monoclonal antibody (1.0 mg/mouse, i.p.) (BioXcell) on 918 Day 0 and 7. Mice were orally gavaged with SM1 or SM3 every third day from Day 1 onwards. 919 Control mice were given isotype control antibody, i.e. rat anti-mouse IgG1 (BioXcell, West 920 Lebanon, NH). Body weights were measured once every two days until euthanasia on Day 18. At 921 euthanasia, blood was collected into BD microtainer (BD Biosciences, San Jose, CA) via cardiac 922 puncture. Hemolysis-free sera were obtained after centrifugation and stored at $-80^{\circ} \mathrm{C}$ until further 923 analysis. Fecal samples were collected and prepared as described previously (Chassaing et al., 924 2012). Fecal lipocalin 2 (Lcn2), serum Lcn2 and serum keratinocyte-derived chemokine (KC) 925 were quantified using Duoset ELISA kits from R\&D Systems (Minneapolis, MN) according to the 926 manufacturer's protocol. Histology scoring for inflammatory damage was performed according to 927 published criteria for colonic inflammation as a consequence of cytokine imbalance (Erben et al., 928 2014). 
929 In vitro co-culture assay using $M$. intestinale. To develop either a broth-based or swarm plate-

930 based co-culture assay the growth kinetics of Muribaculum intestinale (DSM 28989) in Chopped

931 meat carbohydrate broth, PR II (BD, BBL) in an anaerobic chamber, at $37^{\circ} \mathrm{C}\left(\mathrm{O}_{2}=1-2 \%\right)$ was

932 determined (Fig. S7). Early exponential phase cells (OD 600 0.5-0.6) were used to establish the

933 assay. For broth-based assay, $1 \mathrm{~mL}$ of $M$. intestinale cells were mixed to $2 \mu \mathrm{L}$ of SM3/ SM3_18/

934 SM1 grown in LB medium and collected at mid-exponential phase, in a Hungate tube. Cells were

935 collected at different time points $(21 / 24,36,45 / 48 \mathrm{hrs})$, washed in PBS at $5000 \mathrm{rpm}$ for $10 \mathrm{mins}$

936 and total DNA extracted. DNA was resuspended in equal volume of TE buffer.

937 For swarm-plate based assay, 50mL overlay plates containing 30mL LB agar overlaid with 20mL

938 LB swarming agar were prepared. An overnight culture of SM3/SM3_18/SM1 was prepared in

939 LB and spotted, as mentioned before. M. intestinale grown in chopped meat medium was

940 transferred into a bore-well at the center of the plate and incubated at different conditions for 64

941 hours (aerobic, sealed or anaerobic) at $37^{\circ} \mathrm{C}$. For sealed condition, plates were taped carefully

942 using parafilm to maintain anaerobiosis throughout the experiment. Plates incubated in aerobic

943 conditions were maintained at a relative humidity of 50\%. For Divided/Sealed condition, a small

944 Petridish was placed inside a big Petridish and an overlay agar prepared then. The bore well

945 containing $M$. intestinale was stationed in the small Petridish, while the swarming or less swarming

946 strains were spotted on agar present in the big Petridish. Plates were sealed using parafilm, and the

947 presence of an air space between the plates was ensured (please see caricature in Fig. 6). This

948 allowed physical separation of $M$. intestinale from the swarming bacteria, nevertheless maintaining

949 an anaerobic condition in the system. Plates were incubated at $37^{\circ} \mathrm{C}$. At the end of the experiment,

950 well containing cells were collected, washed twice in PBS and then proceeded further for DNA

951 extraction using phenol-chloroform method. DNA was resuspended in equal volume of TE buffer.

952 DNA samples were diluted at least $2 \log$ fold to ascertain DNA copy number/ $\mu \mathrm{L}$ within the range

953 of standard. qPCR analysis was performed using equal volume of each diluted DNA sample and

954 M. intestinale specific primers (Supplementary Table 2). Quantification of amplicon (copy

955 number $/ \mu \mathrm{L}$ ) was calculated based on a standard curve generated using amplicon of interest as

956 template DNA. The specificity of the primers was verified using SM3 genomic DNA in a qPCR

957 reaction. 
958 Statistical analysis. $\mathrm{P}$ values of data were obtained by parametric or non-parametric methods, as

959 indicated in the figure legends, with 95\% confidence interval (CI). Normality (Gaussian

960 distribution) was not assumed and for each dataset this was either tested for or transformed (e.g.,

$961 \log$ normality) to discern whether the data fit a Gaussian distribution. Through visual inspection,

962 sample size assessment, and tests for normality, a determination was made to use a parametric or

963 non-parametric statistical test, as indicated. All statistical tests, except where otherwise indicated,

964 were performed with Graph Pad Prism v.8.2.0; * $\mathrm{P}<0.05$, ** $\mathrm{P}<0.01$, *** $\mathrm{P}<0.001$; ns, not

965 significant. All plots are shown as mean and 95\% CI except where otherwise indicated. 
bioRxiv preprint doi: https://doi.org/10.1101/759886; this version posted October 7, 2020. The copyright holder for this preprint (which was not certified by peer review) is the author/funder, who has granted bioRxiv a license to display the preprint in perpetuity. It is made available under aCC-BY-NC-ND 4.0 International license.

\section{STAR Method Tables}

ST AR Method Table 1 | Bacterial Strains isolated and used in this study

Bacterial strains identified from luminal contents and isolated on swarming agar*

\begin{tabular}{|c|c|c|}
\hline Strain Isolated & Swarming & Source \\
\hline Escherichia coli ${ }^{\#}$ & + & Human IBD \\
\hline Escherichia coli $\#$ & + & Human IBD \\
\hline Escherichia coli & + & Human anal fistula \\
\hline Klebsiella pneumoniae & $+t$ & Human IBD \\
\hline Klebsiella pneumoniae & $-\ddagger$ & Healthy Human \\
\hline Citrobacter koseri & + & Human IBD \\
\hline Morganella morganii & $-\S$ & Human IBD \\
\hline Serratia marcescens & + & Human adenomatous polyp \\
\hline Proteus mirabilis & $+\|$ & Mouse colitis \\
\hline Proteus mirabilis & $+\boldsymbol{\Upsilon}$ & Mouse colitis \\
\hline Enterobacter $s p . \#$ & + & Mouse (DSS colitis) \\
\hline Enterobacter $s p . \#$ & + & Mouse (TNBS colitis) \\
\hline \multicolumn{3}{|l|}{ Bacterial strains used in this study } \\
\hline Organism & Description & Reference \\
\hline Enterobacter sp. SM1 & A clinical isolate from feces of normal mice. & This study \\
\hline$\triangle m o t A \mathrm{SM} 1$ & A flagella motor function abrogated mutant of SM1, motA:.:kan & This study \\
\hline$\triangle f l h E \mathrm{SM} 1$ & A flagella associated gene involved in sw arming, flhE:.:FRT:Kan:FRT & This study \\
\hline HS2B SM1 & $\begin{array}{l}\text { A hyperswarming variant of SM1 generated by serial passage on } \\
\text { swaming agar. }\end{array}$ & This study \\
\hline Enterobacter sp. SM3 & A clinical is olate from feces of DSS-colitis mice. & This study \\
\hline SM3_18 & $\begin{array}{l}\text { A transposon mutant of SM3, putative aerobactin synthesis gene } \\
\qquad \text { iucB:.:Tn:.kan }\end{array}$ & This study \\
\hline SM3_24 & $\begin{array}{l}\text { A transposon mutant of SM3, putative isocitrate/isopropylmalate } \\
\text { dehydrogenase/ADP-ribose pyrophosphate gene:.Tn:.kan }\end{array}$ & This study \\
\hline Serratia marcescens & A clinical isolate from human adenomatous polyp. & This study \\
\hline \multicolumn{3}{|l|}{ Bacillus subtilis } \\
\hline 3610 & A wild-type isolate. & Kearns \& Losick ${ }^{22}$ \\
\hline DS215 & A swarming defective mutant of 3610 , swrA:tet & Kearns et.al ${ }^{23}$ \\
\hline \multicolumn{3}{|l|}{ Serratia marcescens } \\
\hline Db10 & A wild-type isolate. & Pradel et.al ${ }^{24}$ \\
\hline JESM267 & A serrawettin W2 defective mutant of Db10, swrA miniTn5-Sm & Pradel et.al ${ }^{24}$ \\
\hline \multicolumn{3}{|l|}{$\begin{array}{r}\text { Salmonella enterica serovar } \\
\text { Typhimurium }\end{array}$} \\
\hline ATCC 35659 & A wild-type isolate. & \\
\hline$\Delta$ ffil & A swarming deficient mutant of S. enterica, fliL:: FRT. & This study \\
\hline Muribaculum intestinale YL27 & A strict anaerobe isolated from cecal content of mice. & Lagkouvardos et al. \\
\hline \multicolumn{3}{|c|}{$\begin{array}{l}\text { * Human or mouse feces was subject to the swarming assay and any swarm colony detected within } 24 \mathrm{~h} \text { was swabbed for strain identification. In } \\
\text { addition, delayed swamers were classified as negative but their swam edge also yielded single species } \\
\text { † Feces from patient with clinically controlled Crohn's disease with moderate surfactant edge detected at } 74 \mathrm{~h} \\
\text { f Classified as non-swarmer, how ever, a very minimal surfactant edge present at } 24 \mathrm{~h} \text { and no progression thereafter } \\
\text { \$ Feces from patient with clinically controlled Crohn's disease with surfactant edge detected at } 48 \mathrm{~h} \\
\text { II, TI Mouse model: Msh2/-loxPTgfbr2 loxp Villin-cre } \\
\text { \# Also confirmed using Illumina Sequencing (PacBio) }\end{array}$} \\
\hline
\end{tabular}




\section{References}

970

971

972

973

974

975

976

977

978

979

980

981

982

983

984

985

986

987

988

989

990

991

992

993

994

995

996

997

998

999

1000

1001

1002

1003

1004

1005

1006

1007

1008

1009

1010

1011

1012
Alanjary, M., Steinke, K., and Ziemert, N. (2019). AutoMLST: an automated web server for generating multi-locus species trees highlighting natural product potential. Nucleic Acids Res 47, W276-W282.

Alikhan, N.F., Petty, N.K., Ben Zakour, N.L., and Beatson, S.A. (2011). BLAST Ring Image Generator (BRIG): simple prokaryote genome comparisons. BMC Genomics 12, 402.

Allison, C., Emody, L., Coleman, N., and Hughes, C. (1994). The role of swarm cell differentiation and multicellular migration in the uropathogenicity of Proteus mirabilis. J Infect Dis 169, 11551158.

Angelini, T.E., Roper, M., Kolter, R., Weitz, D.A., and Brenner, M.P. (2009). Bacillus subtilis spreads by surfing on waves of surfactant. Proc Natl Acad Sci U S A 106, 18109-18113.

Bankevich, A., Nurk, S., Antipov, D., Gurevich, A.A., Dvorkin, M., Kulikov, A.S., Lesin, V.M., Nikolenko, S.I., Pham, S., Prjibelski, A.D., et al. (2012). SPAdes: a new genome assembly algorithm and its applications to single-cell sequencing. J Comput Biol 19, 455-477.

Barak, J.D., Gorski, L., Liang, A.S., and Narm, K.E. (2009). Previously uncharacterized Salmonella enterica genes required for swarming play a role in seedling colonization. Microbiology 155, 3701-3709.

Be'er, A., and Ariel, G. (2019). A statistical physics view of swarming bacteria. Mov Ecol 7, 9.

Be'er, A., Strain, S.K., Hernandez, R.A., Ben-Jacob, E., and Florin, E.L. (2013). Periodic reversals in Paenibacillus dendritiformis swarming. J Bacteriol 195, 2709-2717.

Bhuwan, M., Lee, H.J., Peng, H.L., and Chang, H.Y. (2012). Histidine-containing phosphotransfer protein-B (HptB) regulates swarming motility through partner-switching system in Pseudomonas aeruginosa PAO1 strain. J Biol Chem 287, 1903-1914.

Bodour, A.A., and Miller-Maier, R.M. (1998). Application of a modified drop-collapse technique for surfactant quantitation and screening of biosurfactant-producing microorganisms. Journal of Microbiological Methods 32, 273-280.

Borton, M.A., Sabag-Daigle, A., Wu, J., Solden, L.M., O'Banion, B.S., Daly, R.A., Wolfe, R.A., Gonzalez, J.F., Wysocki, V.H., Ahmer, B.M.M., et al. (2017). Chemical and pathogen-induced inflammation disrupt the murine intestinal microbiome. Microbiome 5, 47.

Burbank, L., Mohammadi, M., and Roper, M.C. (2015). Siderophore-Mediated Iron Acquisition Influences Motility and Is Required for Full Virulence of the Xylem-Dwelling Bacterial Phytopathogen Pantoea stewartii subsp stewartii. Applied and Environmental Microbiology 81, 139-148.

Butler, M.T., Wang, Q., and Harshey, R.M. (2010). Cell density and mobility protect swarming bacteria against antibiotics. Proc Natl Acad Sci U S A 107, 3776-3781.

Caiazza, N.C., Shanks, R.M., and O'Toole, G.A. (2005). Rhamnolipids modulate swarming motility patterns of Pseudomonas aeruginosa. J Bacteriol 187, 7351-7361.

Caporaso, J.G., Kuczynski, J., Stombaugh, J., Bittinger, K., Bushman, F.D., Costello, E.K., Fierer, N., Pena, A.G., Goodrich, J.K., Gordon, J.I., et al. (2010). QIIME allows analysis of highthroughput community sequencing data. Nat Methods 7, 335-336.

Caporaso, J.G., Lauber, C.L., Walters, W.A., Berg-Lyons, D., Lozupone, C.A., Turnbaugh, P.J., Fierer, N., and Knight, R. (2011). Global patterns of 16S rRNA diversity at a depth of millions of sequences per sample. Proc Natl Acad Sci U S A 108 Suppl 1, 4516-4522. 
1013 Chassaing, B., Aitken, J.D., Malleshappa, M., and Vijay-Kumar, M. (2014a). Dextran sulfate sodium (DSS)-induced colitis in mice. Curr Protoc Immunol 104, Unit 1525.

1015 Chassaing, B., Ley, R.E., and Gewirtz, A.T. (2014b). Intestinal epithelial cell toll-like receptor 5 1016 regulates the intestinal microbiota to prevent low-grade inflammation and metabolic syndrome in mice. Gastroenterology 147, 1363-1377 e1317. preprocessor. Bioinformatics 34, i884-i890.

1020 Cherepanov, P.P., and Wackernagel, W. (1995). Gene disruption in Escherichia coli: TcR and $\mathrm{KmR}$ cassettes with the option of Flp-catalyzed excision of the antibiotic-resistance determinant. Gene 158, 9-14. Typhimurium pathogenicity island 2 is necessary for complete virulence in a mouse model of infectious enterocolitis. Infect Immun 73, 3219-3227.

1026

1027 Colgan, S.P., and Taylor, C.T. (2010). Hypoxia: an alarm signal during intestinal inflammation. Nat Rev Gastroenterol Hepatol 7, 281-287. Jiménez, B., Castro-Alija, M.J., Muñoz-Moreno, M.F., Sánchez, D., Zamora-González, N., BajoGrañeras, R., et al. (2015). Early detection of high oxidative activity in patients with adenomatous intestinal polyps and colorectal adenocarcinoma: myeloperoxidase and oxidized low-density lipoprotein in serum as new markers of oxidative stress in colorectal cancer. Lab Med 46, 123135. Bell, M.E., Hay, A.G., Peterson, D.A., et al. (2013). Innate and adaptive immunity interact to quench microbiome flagellar motility in the gut. Cell Host Microbe 14, 571-581. Datta, S., Costantino, N., and Court, D.L. (2006). A set of recombineering plasmids for gramnegative bacteria. Gene 379, 109-115. D., Hu, P., and Andersen, G.L. (2006). Greengenes, a chimera-checked 16S rRNA gene database and workbench compatible with ARB. Appl Environ Microbiol 72, 5069-5072. tension and related thermodynamic parameters of alcohols using the Traube stalagmometer. European Journal of Physics 26, 1079-1084. Mucosal healing and fibrosis after acute or chronic inflammation in wild type FVB-N mice and C57BL6 procollagen alpha1(I)-promoter-GFP reporter mice. PLoS One 7, e42568. mucins on polyvinylidene difluoride membranes. Anal Chem 84, 8461-8466. M., Siegmund, B., and Kuhl, A.A. (2014). A guide to histomorphological evaluation of intestinal inflammation in mouse models. Int J Clin Exp Pathol 7, 4557-4576.

1058 Cytoscape. F1000Res 5, 1519. 

Cargo Bacteria To Pave the Way in Toxic Environments. mBio 6. Geesala, R., Schanz, W., Biggs, M., Dixit, G., Skurski, J., Gurung, P., Meyerholz, D.K., Elliott, D., Issuree, P.D., and Maretzky, T. (2019). Loss of RHBDF2 results in an early-onset spontaneous murine colitis. J Leukoc Biol 105, 767-781.

1063

Gude, S., Pince, E., Taute, K.M., Seinen, A.B., Shimizu, T.S., and Tans, S.J. (2020). Bacterial coexistence driven by motility and spatial competition. Nature 578, 588-592.

1065

1066 Hall, M., and Beiko, R.G. (2018). 16S rRNA Gene Analysis with QIIME2. Methods Mol Biol 1849, 113-129. flagellated bacteria. Inflamm Regen 37, 23.

Im, E., Riegler, F.M., Pothoulakis, C., and Rhee, S.H. (2012). Elevated lipopolysaccharide in the colon evokes intestinal inflammation, aggravated in immune modulator-impaired mice. Am J Physiol Gastrointest Liver Physiol 303, G490-497.

1073

Jass, J.R. (2003). Hyperplastic-like polyps as precursors of microsatellite-unstable colorectal cancer. Am J Clin Pathol 119, 773-775.

1075

1076

1077 Kearns, D.B. (2010). A field guide to bacterial swarming motility. Nat Rev Microbiol 8, 634-644. Kearns, D.B., and Losick, R. (2005). Cell population heterogeneity during growth of Bacillus subtilis. Genes Dev 19, 3083-3094.

1078

Koster, M., van Klompenburg, W., Bitter, W., Leong, J., and Weisbeek, P. (1994). Role for the outer membrane ferric siderophore receptor PupB in signal transduction across the bacterial cell envelope. EMBO J 13, 2805-2813.

1081

1082 Lagkouvardos, I., Lesker, T.R., Hitch, T.C.A., Galvez, E.J.C., Smit, N., Neuhaus, K., Wang, J., Baines, J.F., Abt, B., Stecher, B., et al. (2019). Sequence and cultivation study of Muribaculaceae reveals novel species, host preference, and functional potential of this yet undescribed family. 1084 Microbiome 7, 28.

1085 Lau, P.C., Sung, C.K., Lee, J.H., Morrison, D.A., and Cvitkovitch, D.G. (2002). PCR ligation 1086 mutagenesis in transformable streptococci: application and efficiency. J Microbiol Methods 49, 193-205.

1088 Lodes, M.J., Cong, Y., Elson, C.O., Mohamath, R., Landers, C.J., Targan, S.R., Fort, M., and 1089 Hershberg, R.M. (2004). Bacterial flagellin is a dominant antigen in Crohn disease. J Clin Invest 113, 1296-1306. in microbial community assembly in a mouse model. ISME J 7, 2116-2125. Morales-Soto, N., Anyan, M.E., Mattingly, A.E., Madukoma, C.S., Harvey, C.W., Alber, M., Deziel, E., Kearns, D.B., and Shrout, J.D. (2015). Preparation, imaging, and quantification of bacterial surface motility assays. J Vis Exp. Invasive Escherichia coli in a Piglet Model of Inflammatory Bowel Disease: Impact on Intestinal Mucosa-associated Microbiota. Front Microbiol 7, 462.

1100 Okada, T., Fukuda, S., Hase, K., Nishiumi, S., Izumi, Y., Yoshida, M., Hagiwara, T., Kawashima, 
1103 Okada, T., Kanda, T., Ueda, N., Ikebuchi, Y., Hashiguchi, K., Nakao, K., and Isomoto, H. (2020).

1104 IL-8 and LYPD8 expression levels are associated with the inflammatory response in the colon of 1105 patients with ulcerative colitis. Biomed Rep 12, 193-198.

1106 Okumura, R., Kurakawa, T., Nakano, T., Kayama, H., Kinoshita, M., Motooka, D., Gotoh, K.,

1107 Kimura, T., Kamiyama, N., Kusu, T., et al. (2016). Lypd8 promotes the segregation of flagellated 1108 microbiota and colonic epithelia. Nature 532, 117-121.

1109 Ormerod, K.L., Wood, D.L., Lachner, N., Gellatly, S.L., Daly, J.N., Parsons, J.D., Dal'Molin, 1110 C.G., Palfreyman, R.W., Nielsen, L.K., Cooper, M.A., et al. (2016). Genomic characterization of the uncultured Bacteroidales family S24-7 inhabiting the guts of homeothermic animals. Microbiome 4, 36. Analysis of Fecal Microbiota and Metabolites in Experimental Colitic Mice during the Inflammatory and Healing Phases. Nutrients 9.

Overhage, J., Bains, M., Brazas, M.D., and Hancock, R.E. (2008). Swarming of Pseudomonas aeruginosa is a complex adaptation leading to increased production of virulence factors and antibiotic resistance. J Bacteriol 190, 2671-2679. Partridge, J.D., and Harshey, R.M. (2013). Swarming: flexible roaming plans. J Bacteriol 195, 909-918.

Perse, M., and Cerar, A. (2012). Dextran sodium sulphate colitis mouse model: traps and tricks. J Biomed Biotechnol 2012, 718617. Beal, C., van Hylckama-Vlieg, J.E., Ballal, S.A., et al. (2014). Gut microbiome composition and function in experimental colitis during active disease and treatment-induced remission. ISME $\mathrm{J} 8$, 1403-1417. Bacterial Genomes by Arbitrarily Primed PCR. Curr Protoc Mol Biol 118, 15 15 11-15 1515. Sasaki, Y., Fukuda, S., Mikam, T., and Hada, R. (2008). Endoscopic quantification of mucosal surface roughness for grading severity of ulcerative colitis. Digestive Endoscopy 20, 67-72. Segata, N., Izard, J., Waldron, L., Gevers, D., Miropolsky, L., Garrett, W.S., and Huttenhower, C. (2011). Metagenomic biomarker discovery and explanation. Genome Biol 12, R60. Effect of Bacillus subtilis PB6, a natural probiotic on colon mucosal inflammation and plasma cytokines levels in inflammatory bowel disease. Indian J Biochem Biophys 46, 79-85. Schwikowski, B., and Ideker, T. (2003). Cytoscape: a software environment for integrated models of biomolecular interaction networks. Genome Res 13, 2498-2504. Singh, V., Yeoh, B.S., Carvalho, F., Gewirtz, A.T., and Vijay-Kumar, M. (2015). Proneness of TLR5 deficient mice to develop colitis is microbiota dependent. Gut Microbes 6, 279-283. Sokolov, A., and Aranson, I.S. (2009). Reduction of viscosity in suspension of swimming bacteria. Phys Rev Lett 103, 148101.

1144 Solovyev, V. (2011). V. Solovyev, A Salamov (2011) Automatic Annotation of Microbial 
Spencer, N.J., Dinning, P.G., Brookes, S.J., and Costa, M. (2016). Insights into the mechanisms underlying colonic motor patterns. J Physiol-London 594, 4099-4116.

Stanton, T.B., and Savage, D.C. (1984). Motility as a factor in bowel colonization by Roseburia cecicola, an obligately anaerobic bacterium from the mouse caecum. J Gen Microbiol 130, 173183.

Suzuki, K., Arumugam, S., Yokoyama, J., Kawauchi, Y., Honda, Y., Sato, H., Aoyagi, Y., Terai, S., Okazaki, K., Suzuki, Y., et al. (2016). Pivotal Role of Carbohydrate Sulfotransferase 15 in Fibrosis and Mucosal Healing in Mouse Colitis. PLoS One 11, e0158967. Tjaden, B. (2015). De novo assembly of bacterial transcriptomes from RNA-seq data. Genome Biol 16, 1.

Tran, H.Q., Ley, R.E., Gewirtz, A.T., and Chassaing, B. (2019). Flagellin-elicited adaptive immunity suppresses flagellated microbiota and vaccinates against chronic inflammatory diseases. Nat Commun 10, 5650. Van Alst, N.E., Picardo, K.F., Iglewski, B.H., and Haidaris, C.G. (2007). Nitrate sensing and metabolism modulate motility, biofilm formation, and virulence in Pseudomonas aeruginosa. Infect Immun 75, 3780-3790. Venkatesh, M., Mukherjee, S., Wang, H., Li, H., Sun, K., Benechet, A.P., Qiu, Z., Maher, L., Redinbo, M.R., Phillips, R.S., et al. (2014). Symbiotic bacterial metabolites regulate gastrointestinal barrier function via the xenobiotic sensor PXR and Toll-like receptor 4. Immunity 41, 296-310.

Volk, J.K., Nystrom, E.E.L., van der Post, S., Abad, B.M., Schroeder, B.O., Johansson, A., Svensson, F., Javerfelt, S., Johansson, M.E.V., Hansson, G.C., et al. (2019). The Nlrp6 inflammasome is not required for baseline colonic inner mucus layer formation or function. $\mathrm{J}$ Exp Med 216, 2602-2618. Biosurfactant Producing Microorganisms. Biosurfactants 672, 1-13. Walters, W., Hyde, E.R., Berg-Lyons, D., Ackermann, G., Humphrey, G., Parada, A., Gilbert, J.A., Jansson, J.K., Caporaso, J.G., Fuhrman, J.A., et al. (2015). Improved Bacterial 16S rRNA Gene (V4 and V4-5) and Fungal Internal Transcribed Spacer Marker Gene Primers for Microbial Community Surveys. mSystems 1 . Whittem, C.G., Williams, A.D., and Williams, C.S. (2010). Murine Colitis modeling using Dextran Sulfate Sodium (DSS). J Vis Exp. Combining quantitative genetic footprinting and trait enrichment analysis to identify fitness determinants of a bacterial pathogen. PLoS Genet 9, e1003716. Wiles, T.J., Schlomann, B.H., Wall, E.S., Betancourt, R., Parthasarathy, R., and Guillemin, K. (2020). Swimming motility of a gut bacterial symbiont promotes resistance to intestinal expulsion and enhances inflammation. PLoS Biol 18, e3000661. contributions. Dis Model Mech 7, 1131-1142. Yilmaz, P., Parfrey, L.W., Yarza, P., Gerken, J., Pruesse, E., Quast, C., Schweer, T., Peplies, J., Ludwig, W., and Glockner, F.O. (2014). The SILVA and "All-species Living Tree Project (LTP)" taxonomic frameworks. Nucleic Acids Res 42, D643-648. algorithms to calculate average nucleotide identity. Antonie Van Leeuwenhoek 110, 1281-1286. 
1193 Young, G.M., Smith, M.J., Minnich, S.A., and Miller, V.L. (1999). The Yersinia enterocolitica 1194 motility master regulatory operon, flhDC, is required for flagellin production, swimming motility, 1195 and swarming motility. J Bacteriol 181, 2823-2833.

1196 Zhang, H.P., Be'er, A., Florin, E.L., and Swinney, H.L. (2010). Collective motion and density 1197 fluctuations in bacterial colonies. Proc Natl Acad Sci U S A 107, 13626-13630. 